This is the accepted version of the article:

Marangon, I.; Ménard-Moyon, C.; Kolosnjaj-Tabi, J.; Béoutis, M.L.; Lartigue, L.; Alloyeau, D.; Pach, E.; Ballesteros, B.; Autret, G.; Ninjbadgar, T.; Brougham, D.F.; Bianco, A.; Gazeau, F.. Covalent functionalization of multi-walled carbon nanotubes with a gadolinium chelate for efficient T1-weighted magnetic resonance imaging. Advanced Functional Materials, (2014). 24. 45: 7173 - . 10.1002/adfm. 201402234.

Available at: https://dx.doi.org/10.1002/adfm.201402234 


\section{Covalent functionalization of multi-walled carbon nanotubes with a gadolinium chelate for efficient T1-weighted magnetic resonance imaging}

Iris Marangon, Cécilia Ménard-Moyon, Jelena Kolosnjaj-Tabi, Marie Lys Béoutis, Lénaic

Lartigue, Damien Alloyeau, Elzbieta Pach, Belén Ballesteros, Gwennhael Autret, Tsedev

Ninjbadgar, Dermot F. Brougham, Alberto Bianco, ${ }^{*}$ Florence Gazeau*

Iris Marangon, Jelena Kolosnjaj-Tabi, Marie Lys Béoutis, Lénaic Lartigue, Florence Gazeau Laboratoire Matières et Systèmes Complexes, UMR 7057 CNRS/Université Paris Diderot, 10 rue Alice Domon et Léonie Duquet, F-75205 Paris Cedex 13, France.

Florence.gazeau@univ-paris-diderot.fr

Cécilia Ménard-Moyon, Alberto Bianco

CNRS, Institut de Biologie Moléculaire et Cellulaire, Laboratoire d'Immunopathologie et Chimie Thérapeutique, UPR 3572, 67000 Strasbourg, France.

a.bianco@ibmc-cnrs.unistra.fr

Jelena Kolosnjaj-Tabi, Gwennhael Autret

Inserm U970, Paris Cardiovascular Research Center-PARCC / Université Paris-Descartes, 56 rue Leblanc, 75015 France.

Damien Alloyeau

Laboratoire Matériaux et Phénomènes Quantiques, UMR 7162 CNRS/Université Paris

Diderot, 10 rue Alice Domon et Léonie Duquet, F-75205 Paris Cedex 13, France.

Elzbieta Pach, Belén Ballesteros

ICN2 - Institut Catala de Nanociencia i Nanotecnologia, Campus UAB, 08193 Bellaterra

(Barcelona), Spain.

Tsedev Ninjbadgar

Faculty of Engineering, Shine Mongol Institute of Technology, Ulaanbaatar 13372, Mongolia.

Dermot F. Brougham

National Institute for Cellular Biotechnology, School of Chemical Sciences, Dublin City University, Dublin 9, Ireland.

Keywords: Carbon nanotubes, Magnetic Resonance Imaging, Contrast agent, Nanomedicine

Given the promise of carbon nanotubes (CNTs) for photothermal therapy, drug delivery, tissue engineering and gene therapy, there is a need for non-invasive imaging methods to monitor 
CNT distribution and fate in the body. In this study non-ionizing whole-body high field magnetic resonance imaging (MRI) is used to follow the distribution of water-dispersible nontoxic functionalized CNTs administrated intravenously to mice. Oxidized CNTs are endowed with positive MRI contrast properties by covalent functionalization with the chelating ligand diethylenetriaminepentaacetic dianhydride (DTPA), followed by chelation to $\mathrm{Gd}^{3+}$. The structural and magnetic properties, MR relaxivities, cellular uptake and application for MRI cell imaging of Gd-CNTs in comparison to the precursor oxidized CNTs are evaluated. Despite the intrinsic $\mathrm{T}_{2}$ contrast of oxidized CNTs internalized in macrophages, the anchoring of paramagnetic gadolinium onto the nanotube sidewall allows for efficient $\mathrm{T}_{1}$ contrast and $\mathrm{MR}$ signal enhancement, which is preserved after CNT internalization by cells. Hence due to their high dispersibility Gd-CNTs have the potential to produce positive contrast in vivo following injection into the bloodstream. The uptake of Gd-CNTs in the liver and spleen is assessed using MRI, while rapid renal clearance of extracellular Gd-CNTs is observed, confirming the evidences of other studies using different imaging modalities. 


\section{Introduction}

Carbon nanotubes (CNTs) have great promise for numerous technological as well as biomedical applications, which arise from their unique architecture and their outstanding mechanical and electronic properties. ${ }^{[1]}$ However, their toxicity and fate in the organism is still a subject of debate. The lack of consensus on a range of toxicology and biodistribution issues regarding CNTs is due, on one part, to the wide heterogeneity of CNTs used in terms of length, diameter, number of walls, purity (e.g. the presence of catalysts), morphology, functionalization, and aggregation state. ${ }^{[2]}$ An additional confounding factor is the limited range of methods available to study CNTs, and more generally carbon-based materials, in biological environments. ${ }^{[3]}$ As pristine CNTs are water insoluble, a variety of chemical strategies have been explored to functionalize the nanotube surface and render them dispersible in aqueous media. ${ }^{[4]}$ The type of surface modification e.g. physical coating as opposed to covalent chemical functionalization, has been shown to play a critical role in the interactions of CNTs with cells, in their behavior in the blood compartment and in their subsequent fate in vivo. ${ }^{[5]}$ In particular, oxidation and shortening of multi-walled carbon nanotubes (MWCNTs) by acid treatment followed by chemical functionalization of the hydrophobic carbon structure with hydrophilic amino groups has led to a new generation of functionalized MWCNTs with high dispersibility, low toxicity, high cellular uptake and favorable pharmacokinetics for medical use. ${ }^{[6]}$ Although functionalized MWCNTs have very promising applications, as they can serve as multifunctional platforms for tumor targeting and drug delivery, ${ }^{[7]}$ therapeutic hyperthermia, ${ }^{[8]}$ transfection of siRNA, ${ }^{[9]}$ and tissue engineering. ${ }^{[10]}$, imaging of MWCNTs in vivo remains a major challenge.

Under controlled conditions imaging of CNTs in biological environments can be achieved using the intrinsic properties of the nanotubes, such as light absorption and scattering, ${ }^{[11]}$ acoustic or photoacoustic properties, ${ }^{[12]}$ Raman signature ${ }^{[13]}$ or electron microscopy. ${ }^{[14]}$ However, with current diagnostic imaging modalities exogenous labels (mainly fluorescent probes $^{[15]}$ and 
radionuclides $^{[16]}$ ) are usually required for efficient non-invasive tracking of CNTs in living animals. $^{[17]}$

Among non-invasive imaging methods, magnetic resonance imaging (MRI) appears particularly advantageous because of its excellent spatial resolution and soft-tissue contrast providing simultaneously anatomic, functional and molecular information. The main advantage of MRI over other modalities, such as fluorescence or nuclear imaging, is its ability to penetrate deep into tissue without employing ionizing radiation. In addition to the enormous, and ongoing, growth in its application in routine clinical practice, MRI has also proven to be a method of choice for molecular imaging, providing cell tracking and direct information on molecular events through the use of smart contrast agents. To date there have been few reports on the use of MRI for visualization of CNTs due to the poor MR contrast generated by CNTs. Recently the magnetic properties of metal impurities present in raw commercially-available singlewalled CNTs (mainly iron and nickel catalysts) were exploited to investigate CNT distribution with hyperpolarized ${ }^{3} \mathrm{He}$ and conventional ${ }^{1} \mathrm{H}$ MRI. ${ }^{[18]}$ However, the intrinsic $\mathrm{T}_{2}$ and $\mathrm{T}_{2}{ }^{*}$ contrast that are exploited in this approach are highly dependent on the quality of purification, on the type of CNTs, and indeed on the aggregation propensity in physiological media; ${ }^{[19]}$ all factors which are not easily controllable. Alternatively, CNTs may be coupled to exogenous contrast agents. These agents may allow both, the $\mathrm{T}_{2}$ contrast and signal reduction, as for example is the case of CNT conjugates with iron oxide superparamagnetic nanoparticles ${ }^{[16 b, 20]}$ or provide $\mathrm{T}_{1}$-based contrast and signal enhancement when the tubes are conjugated to paramagnetic gadolinium ${ }^{[21]}$. The CNT-contrast agent complexes might be either made by loading the tube cavity or by functionalization of their carbon backbone. For the goal of monitoring the behavior of CNTs in vivo, it is important that any exogenous agent does not influence either the CNT biodistribution, or their ability to transport and deliver a therapeutic agent to an identified target. In this regards both external coupling to or internal loading of CNTs with iron oxide nanoparticles may partially modify their fate in vivo, as the organism must degrade inorganic nanoparticles in addition to carbon materials, which are both slow processes. ${ }^{[22]}$ Alternative strategies such as nanoscale confinement of large quantities of Gd within ultra-short SWCNTs through sidewall defects or at the opened ends (so called gadonanotubes), ${ }^{[23]}$ synthesis of SWCNT-Gd oxide complexes by use of $\mathrm{Gd}_{2} \mathrm{O}_{3}$ catalytic nanoparticles ${ }^{[24]}$ or non-covalent adsorption of $\mathrm{Gd}^{3+}$ chelates onto MWCNT surface ${ }^{[25]}$ have been shown to produce agents with very high $\mathrm{r}_{1}$ relaxivity (relaxation enhancement per millimolar concentration of agent). However, non-covalent approaches have the disadvantages 
of saturating the loading capacity of CNTs or strongly reducing the possibility of further functionalization. In addition, most of these Gd-CNT complexes have unknown behavior in vivo, especially after intravenous injection due their poor dispersibility. Only one recent study described intravenous administration of a low dose of SWCNTs, synthesized using gadolinium nanoparticles as catalysts, however an in vivo MRI follow-up was not reported and the fate of gadolinium nanoparticles is not known. ${ }^{[26]}$ In this context, our goal was to produce waterdispersible non-toxic functionalized short MWCNTs suitable for targeting and delivery of drugs $^{[7 \mathrm{a}]}$ with fully characterized MRI contrast properties. The critical point was to endow carbon nanotubes with positive contrast properties without interfering with the nanotube structure and fate. We proposed a covalent grafting of molecular paramagnetic species on the nanotubes.

For this purpose, oxidized MWCNTs were covalently functionalized with the chelating molecule diethylenetriaminepentaacetic dianhydride (DTPA), followed by chelation to $\mathrm{Gd}^{3+}$. As our approach is based on covalent bonding between CNTs and DTPA, which has a high association constant for $\mathrm{Gd}^{3+}$, the chelation of $\mathrm{Gd}^{3+}$ by DTPA/CNTs is strong, resulting in stable Gd-CNT conjugates and thus reducing the risk of $\mathrm{Gd}^{3+}$ release.

The magnetic properties and magnetic resonance relaxivities of the Gd-CNTs in comparison to Ox-CNT showed the paramagnetic contribution of Gd-chelates which led to positive MR contrast. Remarkably these nanotubes were internalized by cells without toxicity and the positive contrast effect was preserved following cell internalization. Finally, the potential for in vivo monitoring of nanotube fate following intravenous administration of Gd-CNTs in mice was assessed demonstrating that MRI can be used as suitable alternative imaging technique to track CNTs in vivo.

\section{Results and discussion}

\subsection{CNT functionalization}

MWCNTs with a diameter of 20-30 nm functionalized with a DTPA derivative were prepared using a four step procedure (Figure 1). Pristine purified MWCNTs were first oxidized in acidic conditions to shorten the tubes to an average length of $\sim 400 \mathrm{~nm}$ and to introduce $\mathrm{COOH}$ groups (Supporting Figure S1). ${ }^{[27]}$ The carboxylic acids were activated using oxalyl chloride. The corresponding acyl chloride groups were then coupled to the DTPA derivative 2 bearing a free 
amine function (Supporting Figure S2). ${ }^{[25,28]}$ The tert-butyl ester moieties were subsequently hydrolyzed using trifluoroacetic acid (TFA). Finally, chelation of $\mathrm{Gd}^{3+}$ was achieved by dispersing the DTPA/CNTs 4 in an aqueous solution of gadolinium(III) perchlorate, followed by dialysis against water to remove free gadolinium. The usual approach to conjugation of molecules with DTPA involves the conversion of one of the four carboxylic acids to an amide functionality by the use of DTPA dianhydride. In addition to the disadvantage of being nonselective, due to the concomitant formation of diamide derivatives, this method results in conjugates with lower chelating capacity for gadolinium. ${ }^{[29]}$ In our case the DTPA derivative 2 has the advantage of bearing five free $\mathrm{COOH}$ groups available for chelation of gadolinium.
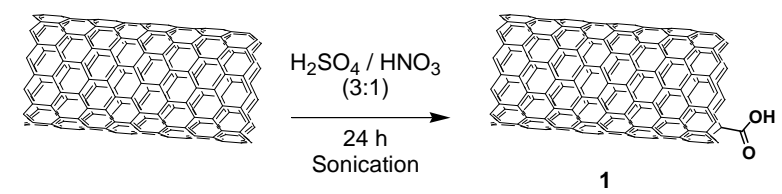

1
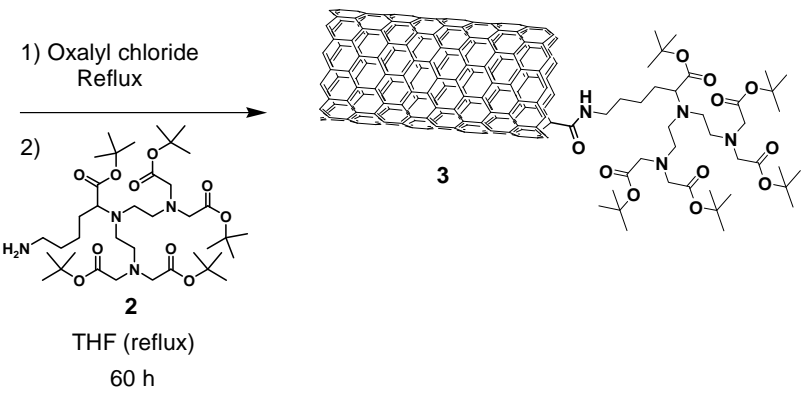

$60 \mathrm{~h}$

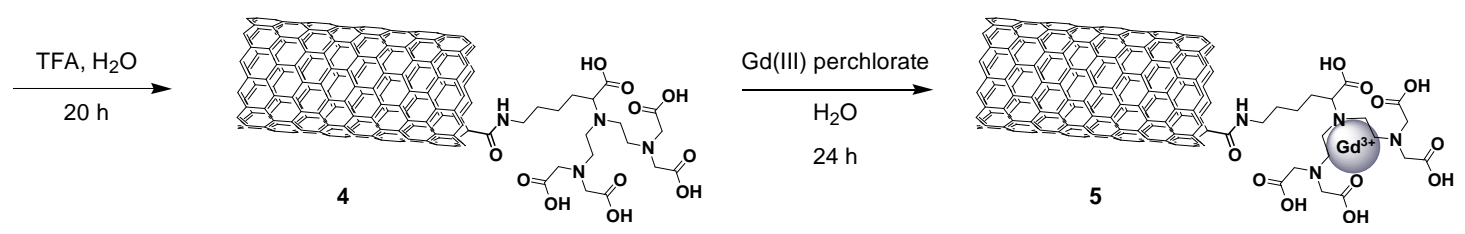

Figure 1: Preparation of Gd-CNTs 5.

The Gd-CNTs were characterized by thermogravimetric analysis (TGA), inductively coupled plasma atomic emission spectrometry (ICP-AES), high resolution transmission electron microscopy (HR-TEM) and scanning transmission electron microscopy (STEM) coupled to energy-dispersive X-ray spectroscopy (EDX). TGA in $\mathrm{N}_{2}$ of pristine MWCNTs, oxidized MWCNTs (ox-CNTs) 1 and DTPA/CNTs 4 showed an increasing weight loss, confirming the efficiency of the functionalization (Figure 2A). The weight loss difference at $500{ }^{\circ} \mathrm{C}$ between ox-CNTs 1 and DTPA/CNTs 4 provides an assessment of the level of functionalization; the 
DTPA loading was estimated $540 \mu \mathrm{mol}$ per gram of CNTs. The level of functionalization was also determined spectrophotometrically using xylenol orange. ${ }^{[30]}$ For this purpose, after chelation of DTPA/CNTs 4 with gadolinium(III) perchlorate and before filtration and dialysis, the free Gd (i.e. not chelated to DTPA/CNTs) was titrated. The dye xylenol orange is used as complexometric indicator of gadolinium as it shows a colour change in the presence of free Gd. This colorimetric test allowed us to determine that the amount of bound gadolinium was 485 $\mu$ mol per gram of CNTs $\mathbf{5}$, which is consistent with the value obtained from TGA. The xylenol orange test was also performed to ensure that no free $\mathrm{Gd}^{3+}$ was present in the Gd-CNT samples. Control reactions were performed using the precursor CNTs (i.e. ox-CNTs 1). In this case, we did not observe a significant complexation of $\mathrm{Gd}^{3+}$ ions by the carboxylic acid functions or the nanotube sidewall. Finally, ICP/AES of Gd-CNTs 5 gave a more precise value of the functionalization degree; $460 \pm 4 \mu \mathrm{mol}$ of gadolinium per gram of nanotubes. Overall, characterization of the CNT conjugates by ICP/MS, TGA, and xylenol orange test is consistent and demonstrate full chelation of $\mathrm{Gd}^{3+}$ by DTPA. TEM analysis of Gd-CNTs 5 show that conditions used for functionalization did not alter the morphology and dispersion of the nanotubes (Supporting Figure S1). The ultrastructure of Gd-CNTs 5, detailed by HR-TEM, revealed structural defects and dislocations within the external graphene layers on which it is likely that the functional groups and Gd-DTPA are anchored (Figure 2B). Gd-CNTs were also characterized using high angle annular dark field (HAADF)-STEM where the intensity in the image is proportional to the atomic number of the elements present in the sample. HAADFSTEM has been shown as a powerful technique to allow the direct visualization of heavyelement bearing organic molecules down to the atomic scale. ${ }^{[31]}$ In the HAADF-STEM images of Gd-CNTs 5, bright dots correspond to the Gd atoms (Figure 2C), while they did not appear in the HAADF-STEM images of DTPA/CNTs $\mathbf{4}$. The presence of gadolinium in Gd-CNTs 5 was confirmed by EDX (Figure 2D), while no Gd was found in the case of DTPA/CNTs 4. 

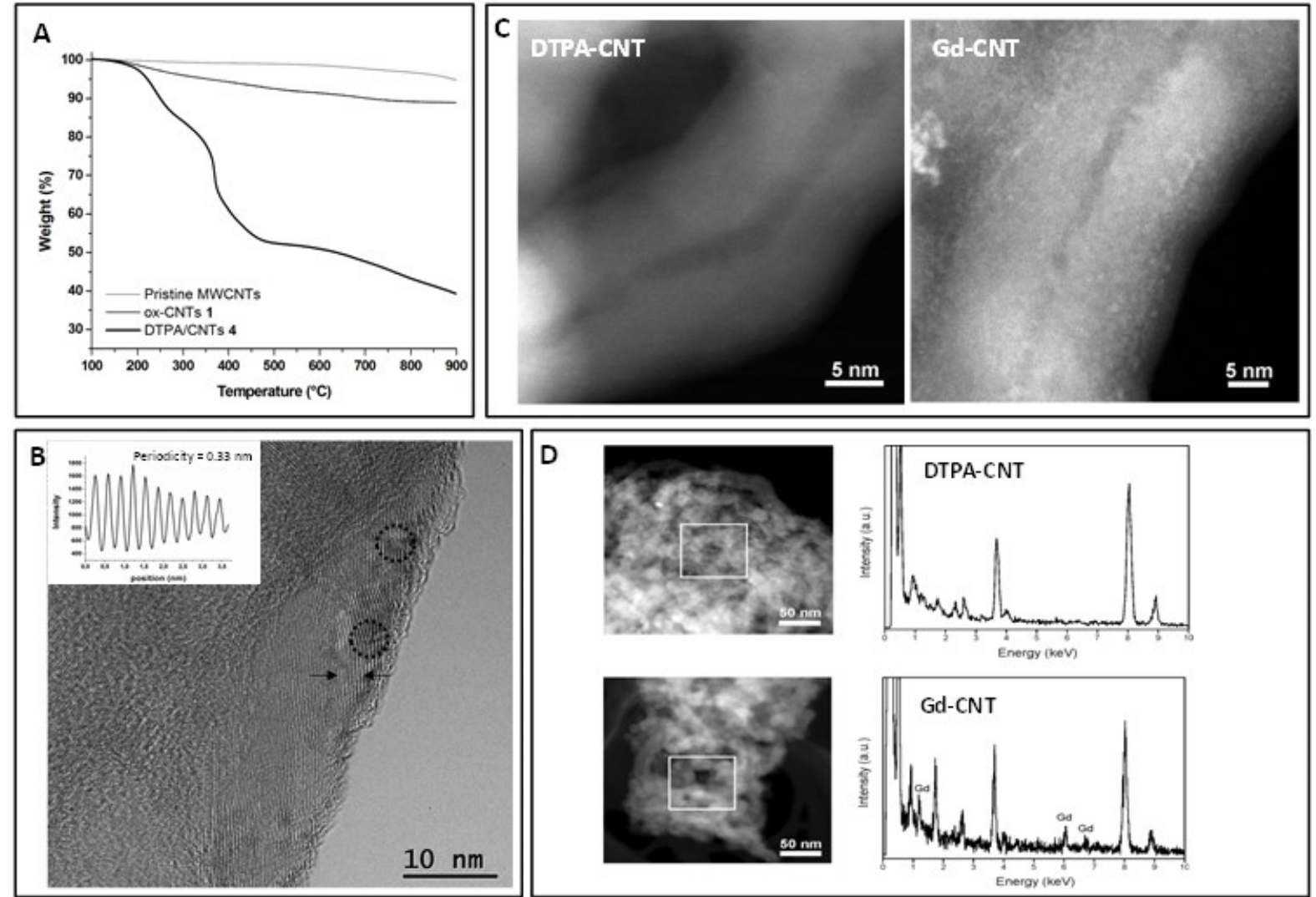

Figure 2 : A) Thermogravimetric analyses of pristine MWCNTs, ox-CNTs 1, and DTPA/CNTs 4 performed in $\mathrm{N}_{2}$ atmosphere. B) Representative HR-TEM micrographs of Gd-CNTs 5 showing structural defects and dislocations within the external graphene layers in which GdDTPA may anchor. Inset shows the interlayer distance between graphene sheets C) HAADF/STEM images of DTPA/CNTs 4 (upper images) and Gd-CNTs 5 (lower images). Groupings of Gd atoms might produce bright spots on HAADF due to the Z-contrast mechanism. D) Comparison of EDX spectrum of DTPA/CNTs 4 and Gd-CNTs 5 confirming the presence of gadolinium.

\subsection{Magnetic characterizations}

Magnetic characterization confirmed the paramagnetic properties of Gd-CNTs 5 in comp arison with the ox-CNT precursor 1. The field-dependent magnetization curve at $5 \mathrm{~K}$ (Figure 3A) was fit using a Brillouin function characteristic of paramagnetic materials, with a $\mathrm{J}$ angular momentum of 3.2. This gives an effective magnetic moment of $6.9 \mu_{\mathrm{B}}$, close to the theoretical magnetic moment of $\mathrm{Gd}^{3+}\left(\mu_{\mathrm{eff}}=7.94 \mu_{\mathrm{B}}, \mathrm{J}=7 / 2\right)$. However, field-cooled (FC) and zero-fieldcooled (ZFC) magnetization measurements (using magnetic fields of 100 and 1000 Oe for the former) revealed an additional ferromagnetic component (Figure 3B) which is added to the 
paramagnetic contribution arising from gadolinium. The FC and ZFC magnetization curves were not strictly superimposable as expected for purely paramagnetic behavior (inset in Figure 3B). Interestingly, the low temperature ferromagnetic component (with a blocking temperature of about $65 \mathrm{~K}$ ) was also displayed by the gadolinium-free control nanotubes (ox-CNTs) (Figure 3C). This suggests that the ferromagnetic contribution arises from residual catalyst impurities (620 ppm of iron and $1283 \mathrm{ppm}$ of nickel), which are probably entrapped into the inner empty cavity of CNTs and were not completely eliminated by the acid treatment, for both ox-CNTs and Gd-CNTs. The chelation of gadolinium into DTPA/CNTs gives rise to the stronger paramagnetic contribution, demonstrated by the linear dependence of magnetization with inverse temperature.
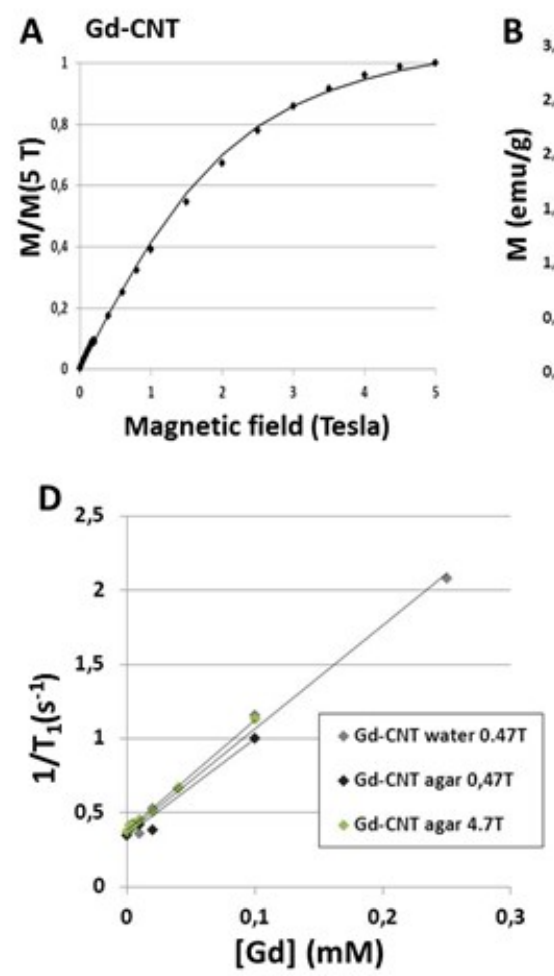
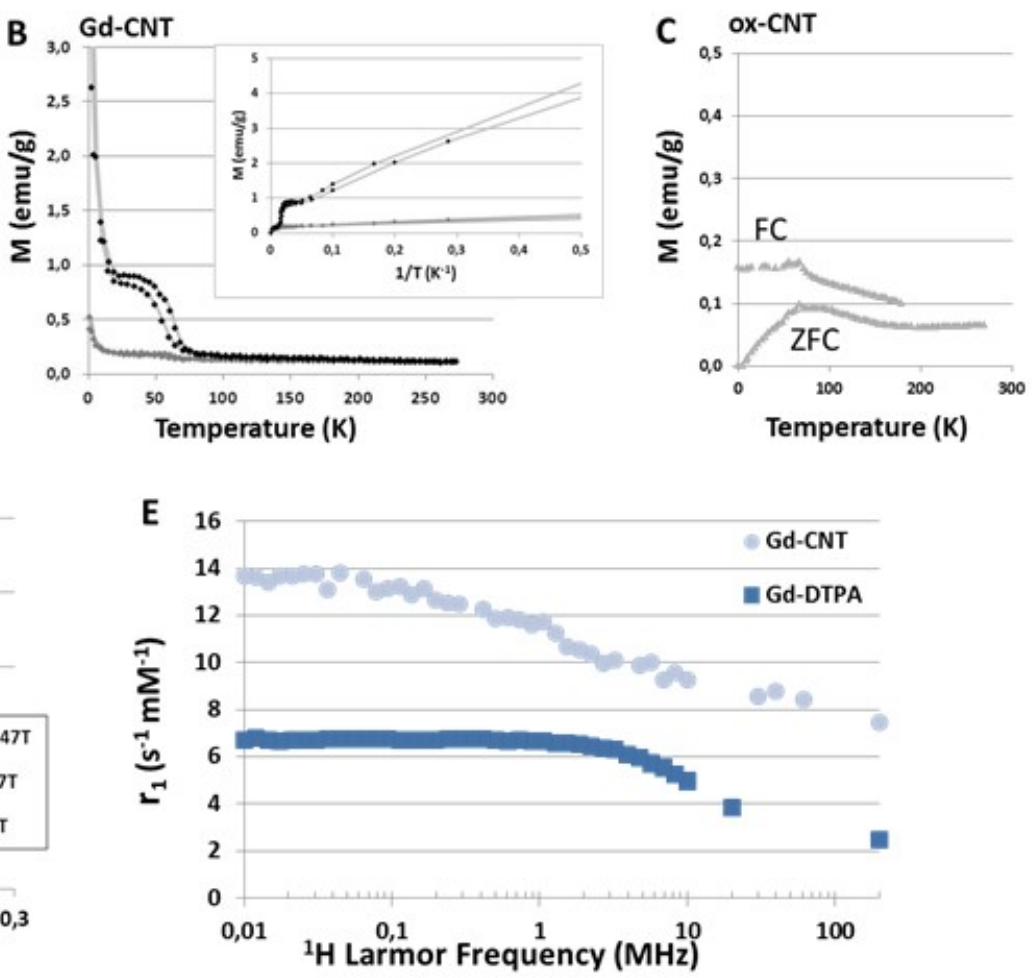

Figure 3 : A) Magnetization curve of Gd-CNTs 5 at $5 \mathrm{~K}$. The solid line indicates the fit using a Brillouin function. B) FC and ZFC temperature dependent magnetization of Gd-CNTs 5 registered at two different magnetic fields: 1000 Oe (black diamonds) and 100 Oe (gray diamonds). The shoulder at low temperature $(<70 \mathrm{~K})$ indicates the presence of a ferromagnetic component. The inset shows the magnetization as a function of inverse temperature. A purely paramagnetic sample would display a linear increase with inverse temperature. C) FC and ZFC magnetization curves (magnetic field $100 \mathrm{Oe}$ ) for the ox-CNTs 1. D) ${ }^{1} \mathrm{H}$ magnetization longitudinal relaxation rate $\left(1 / \mathrm{T}_{1}\right)$ recorded at $25^{\circ} \mathrm{C}$ as a function of $\mathrm{Gd}$ concentration for $\mathrm{Gd}-$ CNTs 5 dispersed in water at $0.47 \mathrm{~T}(20 \mathrm{MHz})$ and in agarose gel at $0.47 \mathrm{~T}(20 \mathrm{MHz})$ and 4.7 
$\mathrm{T}(200 \mathrm{MHz})$. The linear slope yields the relaxivity $\mathrm{r}_{1}$ of Gd-CNTs. E) NMRD profiles ( $\mathrm{r}_{1}$ as a function of ${ }^{1} \mathrm{H}$ Larmor frequency) recorded at $25^{\circ} \mathrm{C}$ for Gd-CNTs 5 in comparison to Gd-DTPA 8.

MRI contrast agents (CAs) act as enhancers of longitudinal and transverse local ${ }^{1} \mathrm{H}$ relaxation rates $R_{1}=1 / T_{1}$ and $R_{2}=1 / T_{2}$. The efficacy of a gadolinium-based $C A$ is measured by its relaxivities $r_{1}$ and $r_{2}$ defined as $r_{i}=\left(R_{i}-R_{i 0}\right) /[G d]$, where $i=1,2$ and $R_{i 0}$ is the endogenous relaxation rate (Figure 3D). Gd-DTPA (Magnevist, Bayer Schering Pharma) is one of the most common clinically approved CAs used in the majority of clinical procedure. ${ }^{[32]}$ It has $r_{1}$ value of $4.1 \mathrm{~s}^{-1} \mathrm{mM}^{-1}$ and $\mathrm{r}_{2}$ of $4.6 \mathrm{~s}^{-1} \mathrm{mM}^{-1}$ at an imaging field of $1.5 \mathrm{~T}$ (or $60 \mathrm{MHz}{ }^{1} \mathrm{H}$ Larmor frequency). A relaxivity ratio $\mathrm{r}_{2} / \mathrm{r}_{1}$ of close to 1 ensures that the $\mathrm{CA}$ acts as a good local signal enhancer (a positive CA), particularly in $\mathrm{T}_{1}$-weighted sequences. Here, the MR properties of Gd-CNTs are compared to those of a Gd-DTPA chelate we synthesized (Supporting Figure S5). The longitudinal relaxivities, $\mathrm{r}$, were measured at $25{ }^{\circ} \mathrm{C}$ over a large range of ${ }^{1} \mathrm{H}$ Larmor frequencies (from 0.01 to $200 \mathrm{MHz}$ ). Interestingly, the resulting nuclear magnetic relaxation dispersion (NMRD) profile of the Gd-CNT suspension is distinctly different from that of nonconjugated Gd-DTPA, with $\mathrm{r}_{1}$ values enhanced by a factor of two at low frequency (Figure 3E). The decrease of $r_{1}$ at clinical fields above $10 \mathrm{MHz}$ is less marked for the Gd-CNTs in comparison to Gd-DTPA. However, the result is a three-fold increase in $\mathrm{r}_{1}$ at $200 \mathrm{MHz}(4.7 \mathrm{~T})$ due to conjugation to CNTs. This effect could be due to an enhancement of the inner-sphere relaxivity mechanism by slowing down the tumbling of Gd-chelates which exhibit fast exchange of the coordinated water molecules. This characteristic effect has been achieved before by binding Gd ions to objects of increasing size from macromolecules to proteins or nanoparticles. ${ }^{[33]}$ In our case, the nearly one-dimensional structure of functionalized CNTs together with their high dispersibility, highly hydrated surface and high permeability to water may favor water exchange, while increasing the correlation time for tumbling of the whole conjugate. Importantly the huge igeneratncrease of low field relaxivity demonstrated by non- 
covalent confinement of Gd-complexes or gadolinium oxide into nanosystems permeable to water, such as zeolites, apoferritin, silicon microparticles and single-walled carbon nanotubes was not observed. ${ }^{[23 a, 34]}$ This is consistent with an external covalent coupling of wateraccessible Gd-chelates at the surface of the oxidized nanotubes. It should also be noted that we did not observe variations of relaxivity with CNT concentration, as previously reported. ${ }^{[25]}$ All relaxation rates showed linear variation with $\mathrm{CNT}$ and $\mathrm{Gd}$ concentration with identical $\mathrm{r}_{1}$ relaxivities whether CNTs have been dispersed in water or further immobilized in agarose gel (Figure 3D). All relaxation rates showed linear variation with CNT and Gd concentration, with identical $\mathrm{r}_{1}$ values obtained irrespective of whether the Gd-CNTs were dispersed in water or immobilized in agarose gel (Figure 3D). The former observation confirms both the remarkably high dispersibility of the functionalized CNTs and their colloidal stability in water suspension over the concentration range studied. The latter suggests that further increases in the overall tumbling correlation time (in agarose) have no effect, and so this is no longer the r1-limiting process. Once bound to the CNT it seems that the timescale for local dynamics about the chelate determines the relaxivity.

Transverse relaxivities $\mathrm{r}_{2}$ were measured in the frequency range between $20 \mathrm{MHz}(0.47 \mathrm{~T})$ and $200 \mathrm{MHz}(4.7 \mathrm{~T})$. The values of $\mathrm{r}_{2}$ of Gd-CNTs and Gd-DTPA decreased from $13.0 \mathrm{~s}^{-1} \mathrm{mM}^{-1}$ and 5.40 at $20 \mathrm{MHz}$ to $5.22 \mathrm{~s}^{-1} \mathrm{mM}^{-1}$ and $4.07 \mathrm{~s}^{-1} \mathrm{mM}^{-1}$ at $200 \mathrm{MHz}$, respectively. This change corresponds to an evolution of the $\mathrm{r}_{2} / \mathrm{r}_{1}$ ratio from 1.8 to 0.7 for Gd-CNTs and from 1.42 to 1.40 for Gd-DTPA when the imaging field is increased from 0.47 to $4.7 \mathrm{~T}$. Hence, in terms of $\mathrm{r}_{2} / \mathrm{r}_{1}$ ratio, the efficacy as a positive CA is enhanced for Gd-CNTs on increasing the field, in comparison to Gd-DTPA. Therefore, for the in vitro and in vivo evaluation of Gd-CNTs, an imaging field of $4.7 \mathrm{~T}$ was used.

\subsection{Cellular uptake and MRI cell imaging}


As intrinsic MR contrast highly depends on tissue structure and water dynamics, the ability of a CA to modify the ${ }^{1} \mathrm{H}$ signal is strongly affected by the local environment and by $\mathrm{CA}$ organization in this environment. During their odyssey through the organism, CNTs are likely internalized by cells, and most probably by cells of the reticulo-endothelial system (resident macrophages or circulating phagocytic cells). However, the magnetic properties of MRI contrast agents are deeply affected by cellular internalization and intracellular compartmentalization. For instance it is well-known that there is a drastic reduction of the longitudinal relaxivity of iron oxide nanoparticles following cellular uptake. ${ }^{[35]}$ Similar effects were observed for gadolinium oxide nanoparticles depending on the internalized dose ${ }^{[36]}$ and for a macromolecular Gd(III)-based probe ${ }^{[37]}$ depending on its intracellular localization. ${ }^{[38]}$ This so-called intracellular "quenching" effect tends to enhance $\mathrm{r}_{2} / \mathrm{r}_{1}$ ratio, in such manner that the generation of positive contrast by labeled cells becomes challenging. Regarding CNTs, it is not clear how $\mathrm{r}_{1}$ could be modified by cell confinement and how intrinsic properties of precursor ox-CNTs could affect transverse and longitudinal relaxation in that environment. It was reported that, despite a very high $\mathrm{r}_{1}$ value in suspension, gadolinium-loaded SWCNTs failed to induce positive contrast when internalized by murine macrophages and were subsequently detected with a negative signal on $\mathrm{T}_{2}$ or $\mathrm{T}_{2}{ }^{*}$-weighted sequence. ${ }^{[21 \mathrm{c}]}$ Likewise, CNTs that were free of any gadolinium label ${ }^{[18 b]}$ were found to induce ${ }^{1} \mathrm{H}$ signal suppression in mouse liver. These confusing results call for a thorough examination of MR properties depending on cellular uptake and on the intrinsic magnetic properties of CNTs. In this work, RAW 264.7 mouse macrophages were used as a cell model to study Gd-CNT uptake in vitro. The cells were incubated for 24 hours with Gd-CNTs in the concentration range of $0-100 \mu \mathrm{g} / \mathrm{mL}$. Cell morphology and adherence on culture flasks was not affected by CNT labeling as shown by optical microscopy (Figure 4A). Cellular metabolic activity, assessed by the Alamar blue test, revealed a slight decrease in comparison to non-labelled cells at the highest concentration tested $(100 \mu \mathrm{g} / \mathrm{mL})$ (see Supporting Figure S4). TEM confirmed the intracellular uptake of CNTs; 
indeed elongated hollow structures were easily identifiable within the intracellular organelles (Figure 4B). Most nanotubes were assembled as large bundles within vesicular membranes, only a small fraction could be observed isolated within the cytoplasm. This suggests an active process of cellular internalization, involving membrane trafficking and dynamic compartimentalization within lysosomal storage organelles. The dose dependent CNT uptake by macrophages was assessed by imaging flow cytometry (ImageStream $\left.{ }^{\circledR}\right) .{ }^{[11 b]}$ This technique combines the statistical power of high throughput flow cytometry with imaging of each analyzed cell. ${ }^{[11 b, 39]}$ As illustrated in Figure 5A, Gd-CNT-labeled cells exhibit dark spots on bright field images that were not observed in non-labeled cells. These dark spots colocalized with high intensity spots on the dark field image, corresponding to areas of CNT accumulation that strongly absorb and scatter the light. The biparametric dot plot representing the mean intensity on dark field versus the mean pixel intensity on bright field (Figure 5B) clearly shows that both parameters increase (in absolute value) with increasing concentration of CNTs in the incubation medium. This indicates a dose dependent CNT uptake for the entire cell population, which tends to saturate at the highest concentration. Similar results were obtained for both GdCNTs and ox-CNTs. 


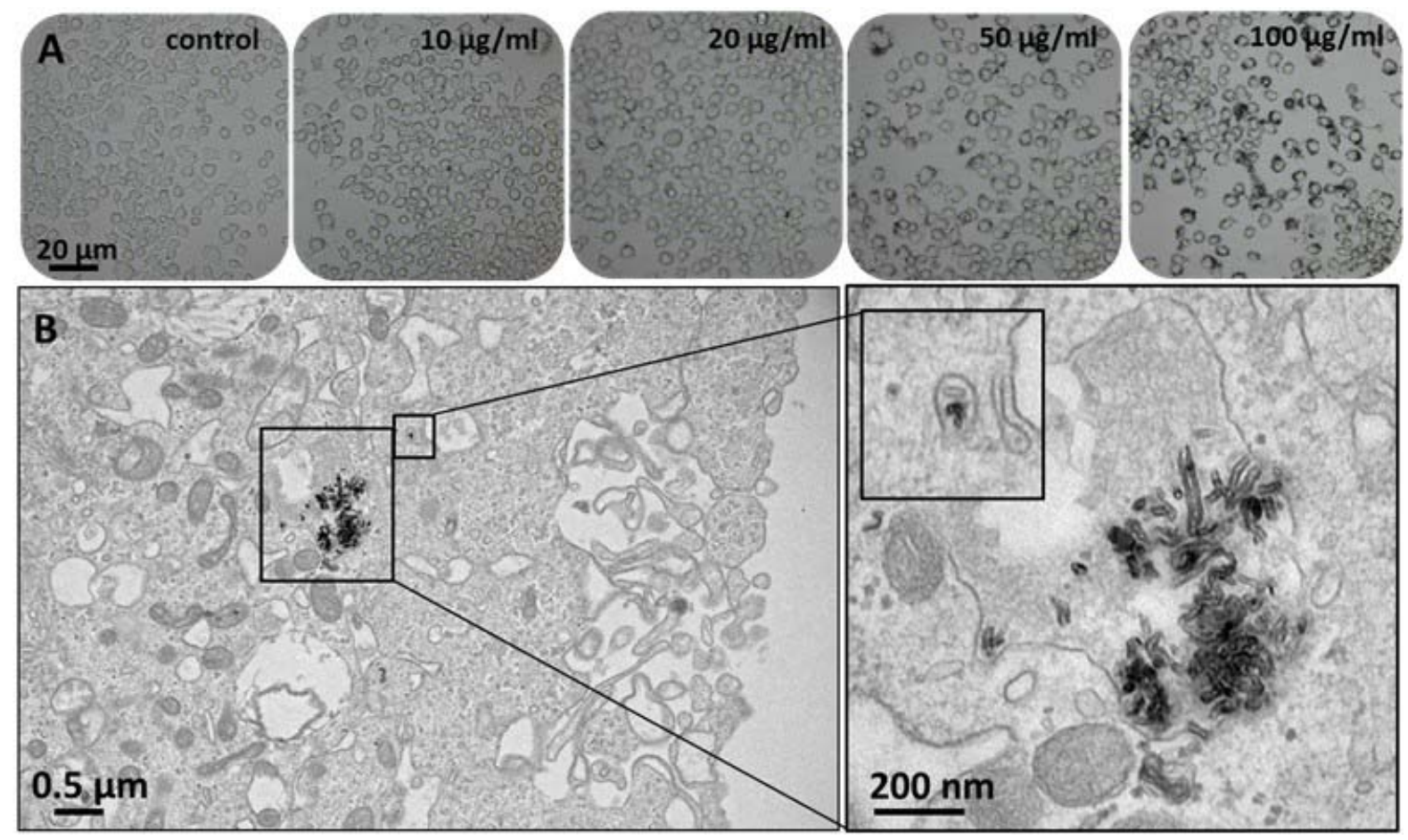

Figure 4 : A) Optical micrographs of RAW 264.7 mouse macrophages after $20 \mathrm{~h}$ incubation with Gd-CNTs 5 at concentrations of 10, 20, 50 and $100 \mu \mathrm{g} / \mathrm{mL}$. B) Electron micrographs of RAW cells incubated with $20 \mu \mathrm{g} / \mathrm{mL}$ of Gd-CNTs 5 for $20 \mathrm{~h}$. Isolated Gd-CNTs and bundles of Gd-CNTs can be observed inside membrane-bound endosomes.

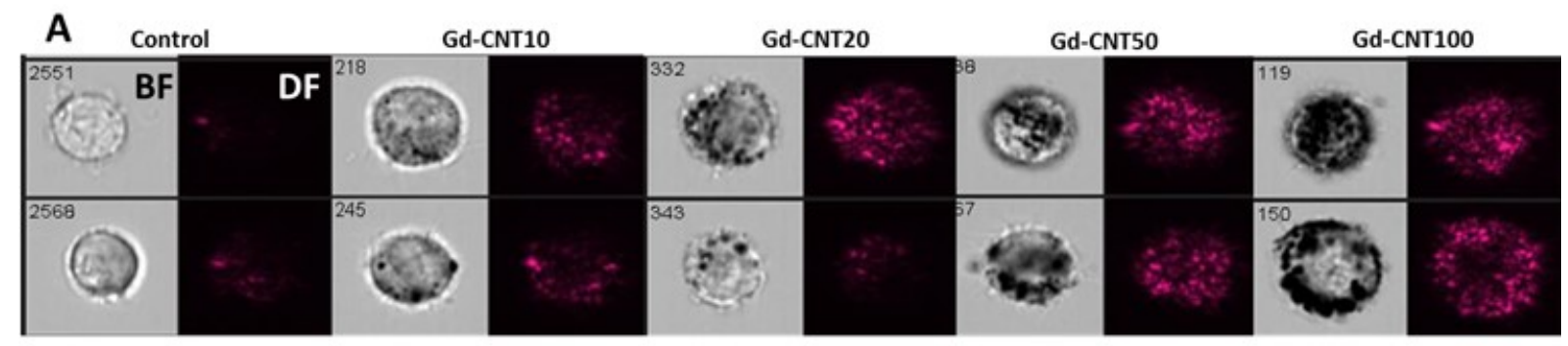

B

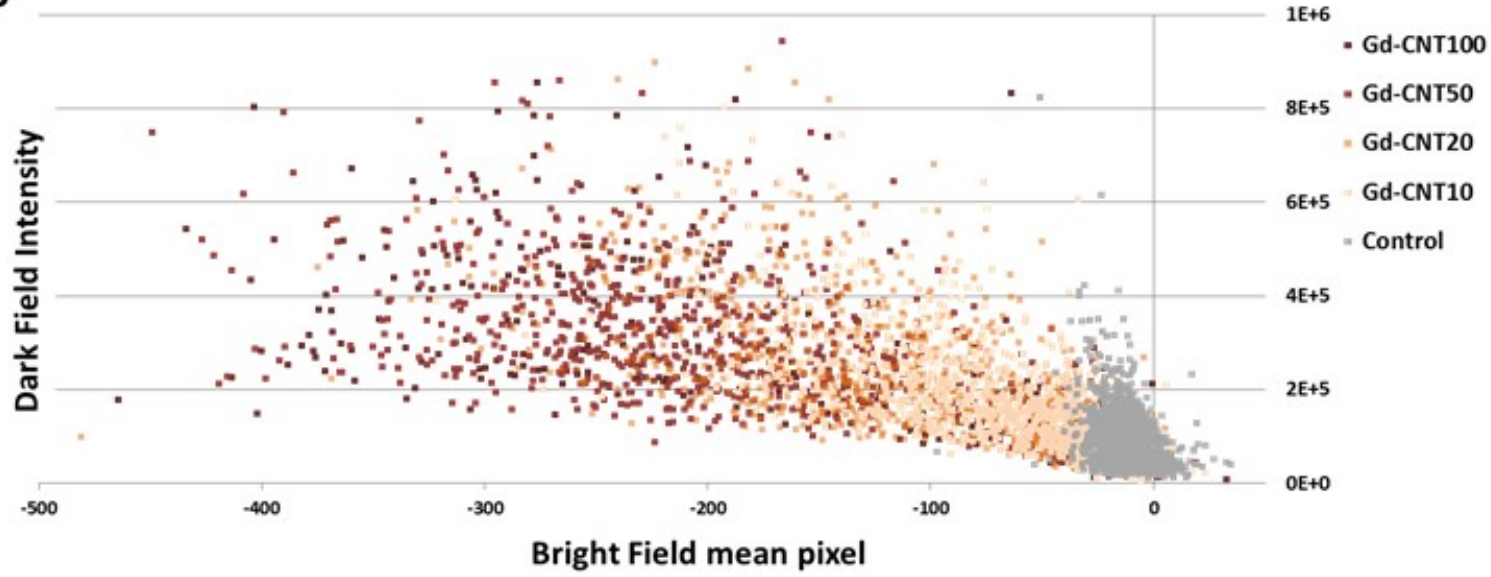

Figure 5 : Imaging flow cytometry analysis of Gd-CNT uptake by RAW 264.7 macrophages. A) Representative bright field and corresponding dark field images of cells (among 10000 ) acquired with ImageStream ${ }^{\circledR}$ for different Gd-CNT incubation conditions. Black spots on bright field images corresponding to bright spots on dark field images are indicative of CNT 
uptake. B) Relative quantification of Gd-CNT uptake on biparametric dot plot of dark field intensity versus mean pixel intensity on bright field image.

Subsequently the MR contrast properties of cells labeled with increasing concentration of GdCNTs or ox-CNTs and dispersed in agarose gels mimicking the cell density of tissue $\left(7 \times 10^{6}\right.$ cells in $70 \mu \mathrm{L}$ of gel) were examined. MR images were acquired using a spin echo sequence, while varying the echo time $T_{E}$ or repetition time $T_{R}$ in order to determine $T_{1}$ and $T_{2}$ values and the optimal conditions for $\mathrm{T}_{1}$ - or $\mathrm{T}_{2}$-weighting. Representative $\mathrm{T}_{1}$-weighted images in Figure 6A show a dose-dependent signal increase in the macrophages labeled with Gd-CNTs in comparison to the non-labeled cells or to the cells labeled with ox-CNTs. The signal increase was nearly two-fold for the highest Gd-CNT concentration with respect to non-labeled cells. Conversely, in T2-weighted images, the macrophages labeled with Gd-CNTs display decreased MR signal intensity with increasing CNT concentration (Figure 6A). Hence, the Gd-CNTs act as a bimodal contrast agent for cell imaging, generating positive contrast under $\mathrm{T}_{1}$-weighted and negative contrast under $\mathrm{T}_{2}$-weighted imaging conditions. By comparison, no signal change was observed for macrophages labeled with ox-CNTs. The quantitative determination of relaxation rates $\left(1 / \mathrm{T}_{\mathrm{i}}\right.$ expressed in $\left.\mathrm{s}^{-1}, \mathrm{i}=1,2\right)$ reveals a linear increase of $1 / \mathrm{T}_{1}$ with Gd-CNT concentration in macrophages, whereas no variation was observed after labeling with control ox-CNTs (Figure 6B). This shows that Gd-DTPA chelates covalently coupled to CNTs can still be effective as $\mathrm{T}_{1}$-contrast agents when internalized in cells. Regarding the transverse relaxation, the analysis is more complex. In comparison to cell-free agarose, the high density of cells induces a 3 -fold increase in transverse relaxation time $1 / \mathrm{T}_{2}$ (Figure 6B). This observation is consistent with relaxation dynamics in tissue, which is accelerated in comparison to water ${ }^{[40]}$ More surprisingly, $1 / \mathrm{T}_{2}$ also increased with the concentration of CNTs in cells, regardless of whether they were labeled with Gd-CNTs or ox-CNTs. The $\mathrm{T}_{2}$ effect appears as an intrinsic characteristic of CNTs, not of the complexed gadolinium probe. In summary 
covalent grafting of Gd-DTPA induces an efficient intracellular $\mathrm{T}_{1}$ reduction that depends on CNT cellular uptake. Despite the associated $\mathrm{T}_{2}$ reduction in labeled cells arising from the nanotubes, positive cell contrast due to the paramagnetic label can be observed when appropriate $\mathrm{T}_{1}$-weighting is applied.

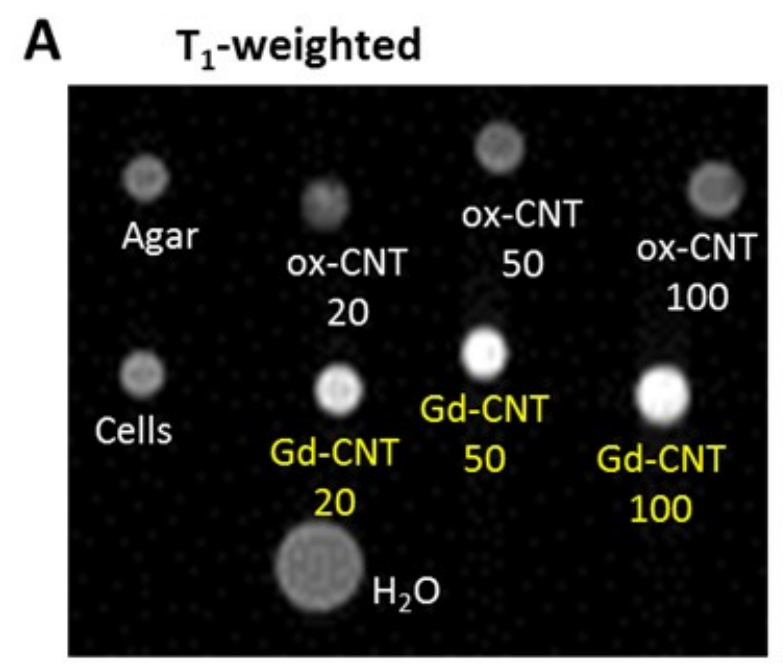

\section{$T_{2}$-weighted}
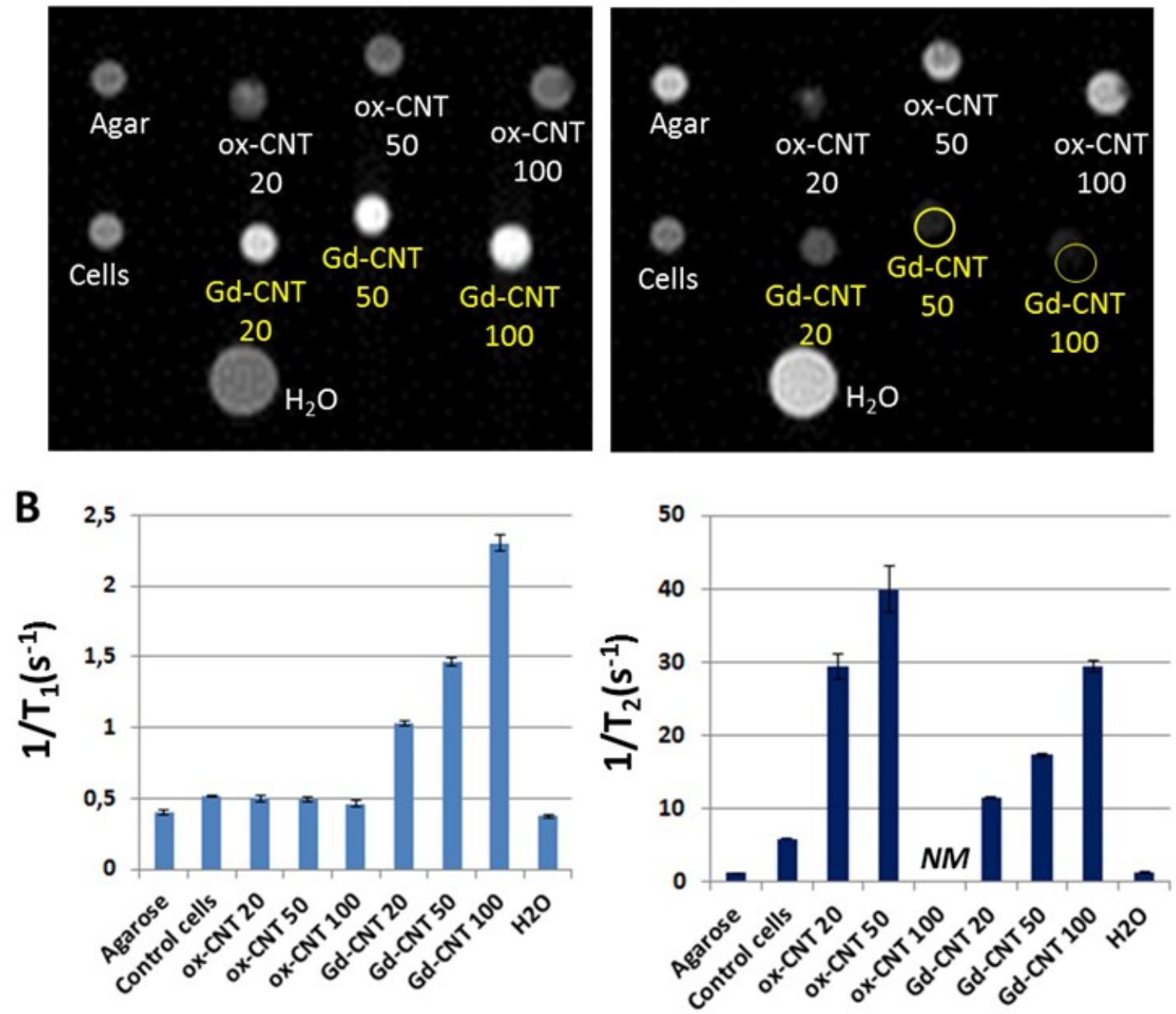

Figure 6 : A) $\mathrm{T}_{1}$-weighted and $\mathrm{T}_{2}$-weighted fast spin echo MR images of compacted cells mimicking tissue $(70 \mu \mathrm{L})$ labeled with different concentrations $(10,20,50$ and $100 \mu \mathrm{g} / \mathrm{mL})$ of Gd-CNTs 5 or ox-CNTs $\mathbf{1}$. For comparison, images of agarose gel alone (agar), water alone or non-labeled cells (cells) are also displayed. $\mathrm{T}_{\mathrm{E}} / \mathrm{T}_{\mathrm{R}}$ are respectively $445 / 12 \mathrm{~ms}$ for $\mathrm{T}_{1}$-weighted sequence and 4500/95 $\mathrm{ms}$ for $\mathrm{T}_{2}$-weighted sequence. B) Longitudinal, 1/ $\mathrm{T}_{1}$, and transverse, $1 / \mathrm{T}_{2}$, relaxation rates of compacted cells for different labeling conditions (NM: not measurable). 


\subsection{In vivo imaging}

The next step was to evaluate the use of Gd-CNTs in vivo to monitor the organ biodistribution of functionalized nanotubes. Gd-CNTs were injected intravenously to mice at two different doses (50 $\mu \mathrm{g}$ and $250 \mu \mathrm{g}$ corresponding to 2.5 and $12.5 \mathrm{mg} / \mathrm{kg}$, respectively) and monitored over 5 hours following injection. A $\mathrm{T}_{1}$-weighted sequence (fast spin echo) was applied since it offered the best contrast-to-noise ratio after Gd-CNT administration. Following the injection of the low dose of Gd-CNTs, significant signal changes in liver, spleen, kidneys and bladder were not observed. However a five-fold increase in Gd-CNT dose $(250 \mu \mathrm{g}$ corresponding to a gadolinium dose of $5.75 \mu \mathrm{mol} / \mathrm{kg}$ body weight) yielded a clear signal enhancement in liver, spleen and to a much larger extent in the bladder, where signal enhancement in excess of $+250 \%$ was obtained (Figure 7). While the contrast enhancement decreased after 2 hours in the bladder (Figure 7C), it was slightly increased over time in liver reaching a value of $+27 \%$ after 320 min (Figure 7AB). In spleen the signal reached a maximum after two hours $(+16 \%)$ and then decreased to close to its initial value $(+3 \%)$. Histological specimens of hepatic and splenic tissues confirmed the presence of nanotubes with a spotty homogenous distribution in liver and in the red pulp periphery of the spleen (Figure 8 and Supporting Figures S5\&S6). 
A

$\%$ Contrast enhancement

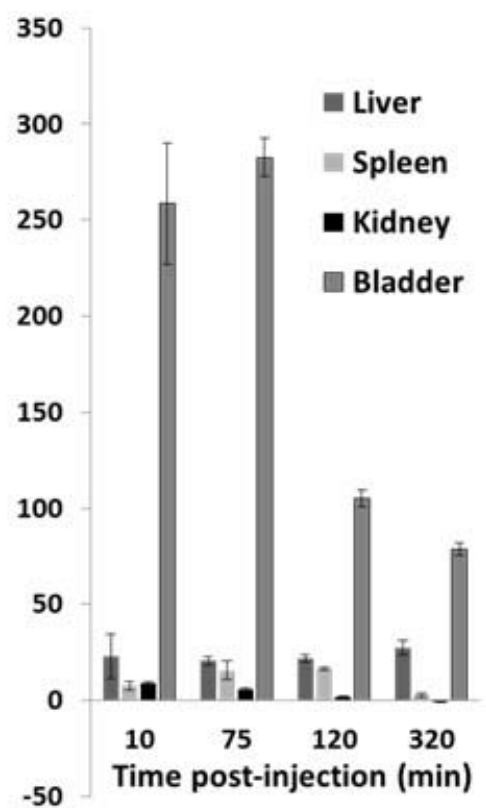

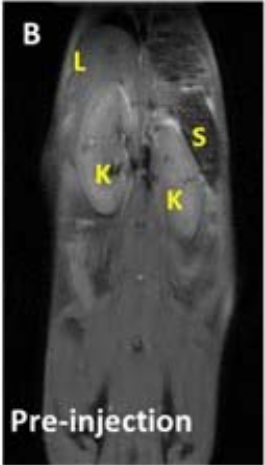
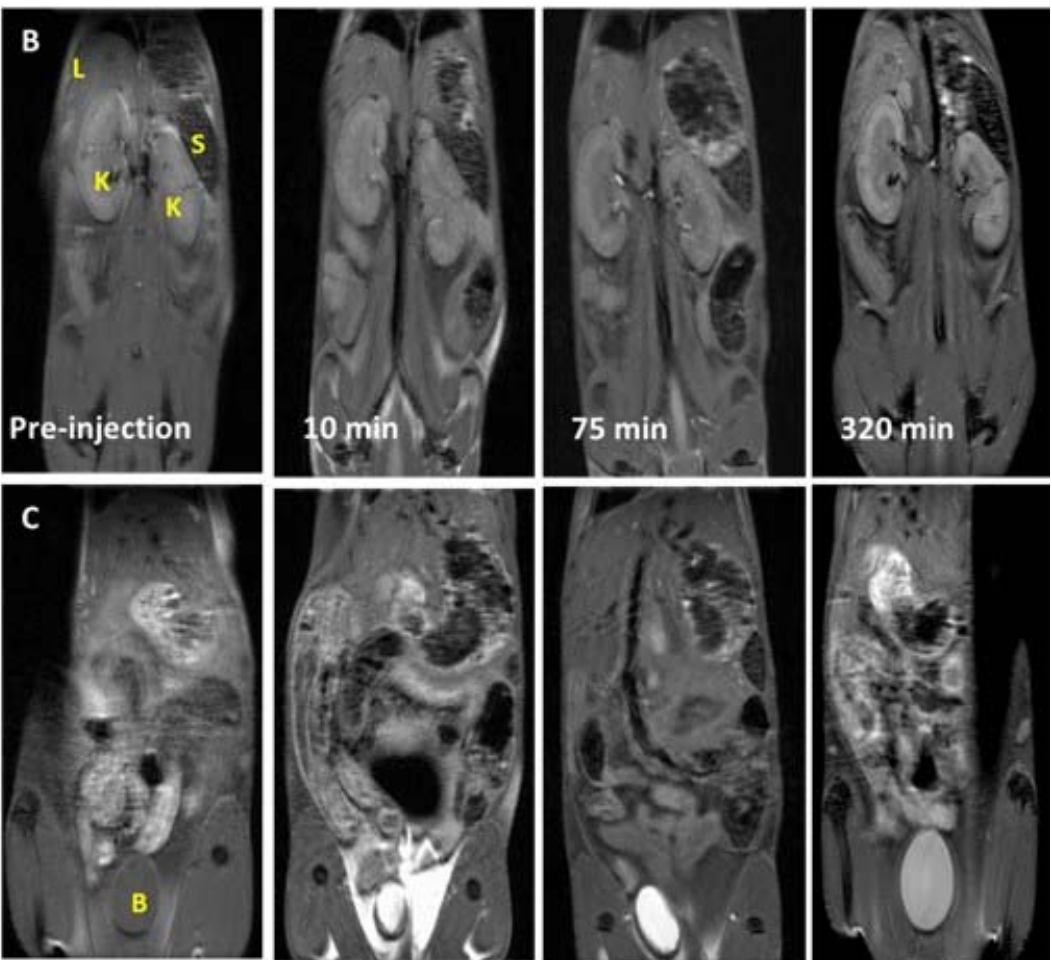

Figure 7: A) MRI contrast enhancement in liver, spleen, kidneys and bladder following intravenous injection of Gd-CNT $5(5 \mathrm{mg} / \mathrm{mL}, 100 \mu \mathrm{L})$ in comparison to the signal prior injection. Standard deviations are deduced from three independent measurements. B-C) Example of MR slices showing liver $(L)$, spleen $(S)$ and kidney $(K)(\mathbf{B})$ or bladder $(B)(\mathbf{C})$ at different-time points before and after injection of Gd-CNTs. A fast spin echo RARE $\mathrm{T}_{1}$ weighted sequence was used with $\mathrm{T}_{\mathrm{E}} / \mathrm{T}_{\mathrm{R}}=445 / 12 \mathrm{~ms}$.
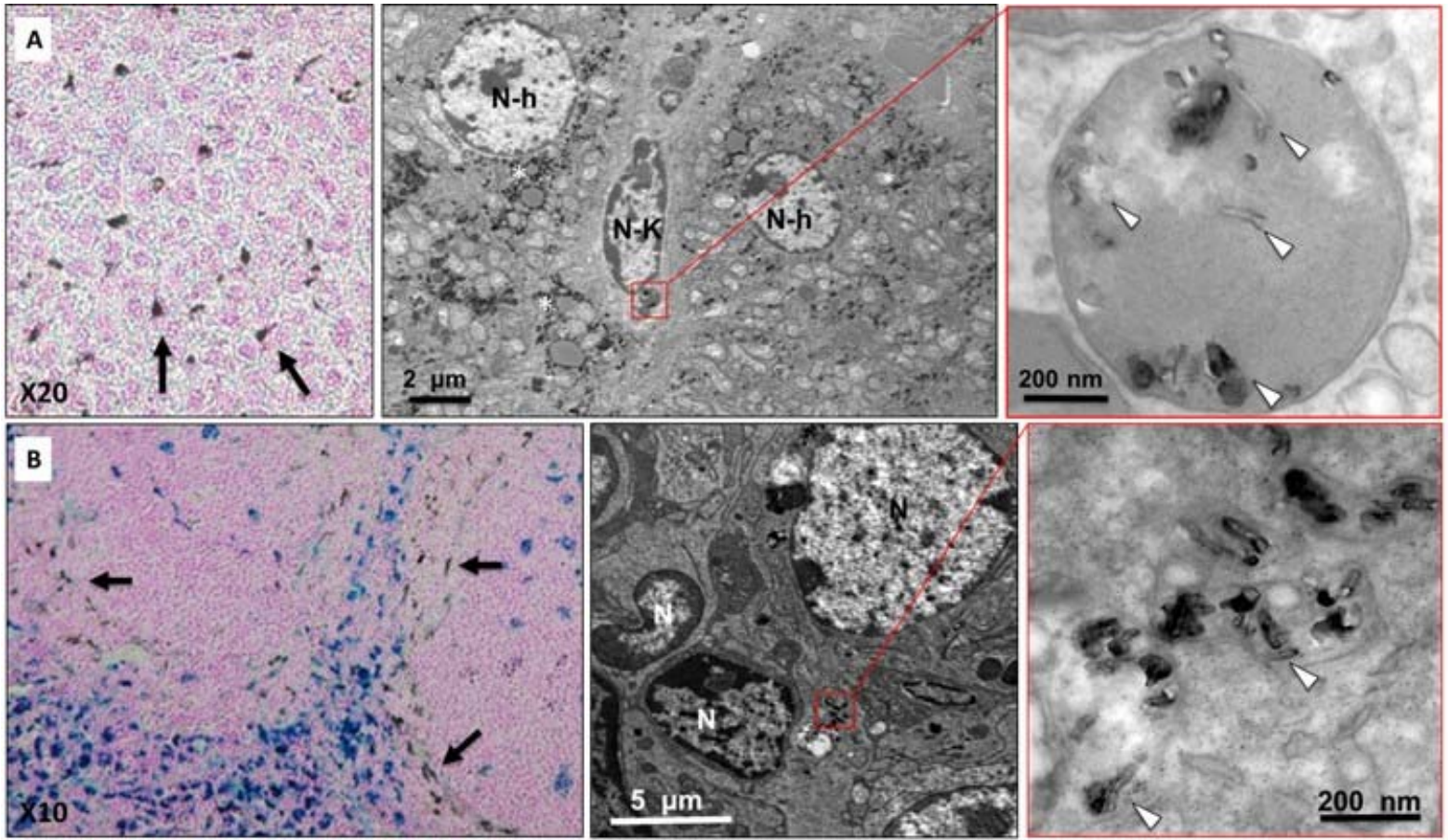

Figure 8 : Histological (left) and transmission electron microscopy (TEM) (right) micrographs of liver (A) and spleen (B) 6 hours after injection of Gd-CNTs 5. A) Note the homogenous distribution of Gd-CNT aggregates (brown spots) within the liver (histological slices were 
stained with Nuclear Fast Red, Mag x20). TEM shows that Gd-CNTs are mainly localized in endosomes within Kupffer cells (N-h: nuclei of hepatocytes, N-K: nucleus of the Kupffer cell, asterisks: glycogen, white arrowhead: endosome containing nanotubes and its magnified view in the inset. White arrows point to nanotubes). B) Black arrows point to Gd-CNT aggregates (brown spots on histology (left)) within the spleen (Nuclear Fast Red and Pearls staining, which reveals endogenous iron storage ferritin (blue spots), Mag x10). Aggregates are mainly localized in the periphery or, in case of smaller aggregates, in the center of the white pulp. TEM micrographs show Gd-CNTs localized in endosomes of phagocytic cells (N: nuclei, red square: endosome containing nanotubes and its magnified view on the right).
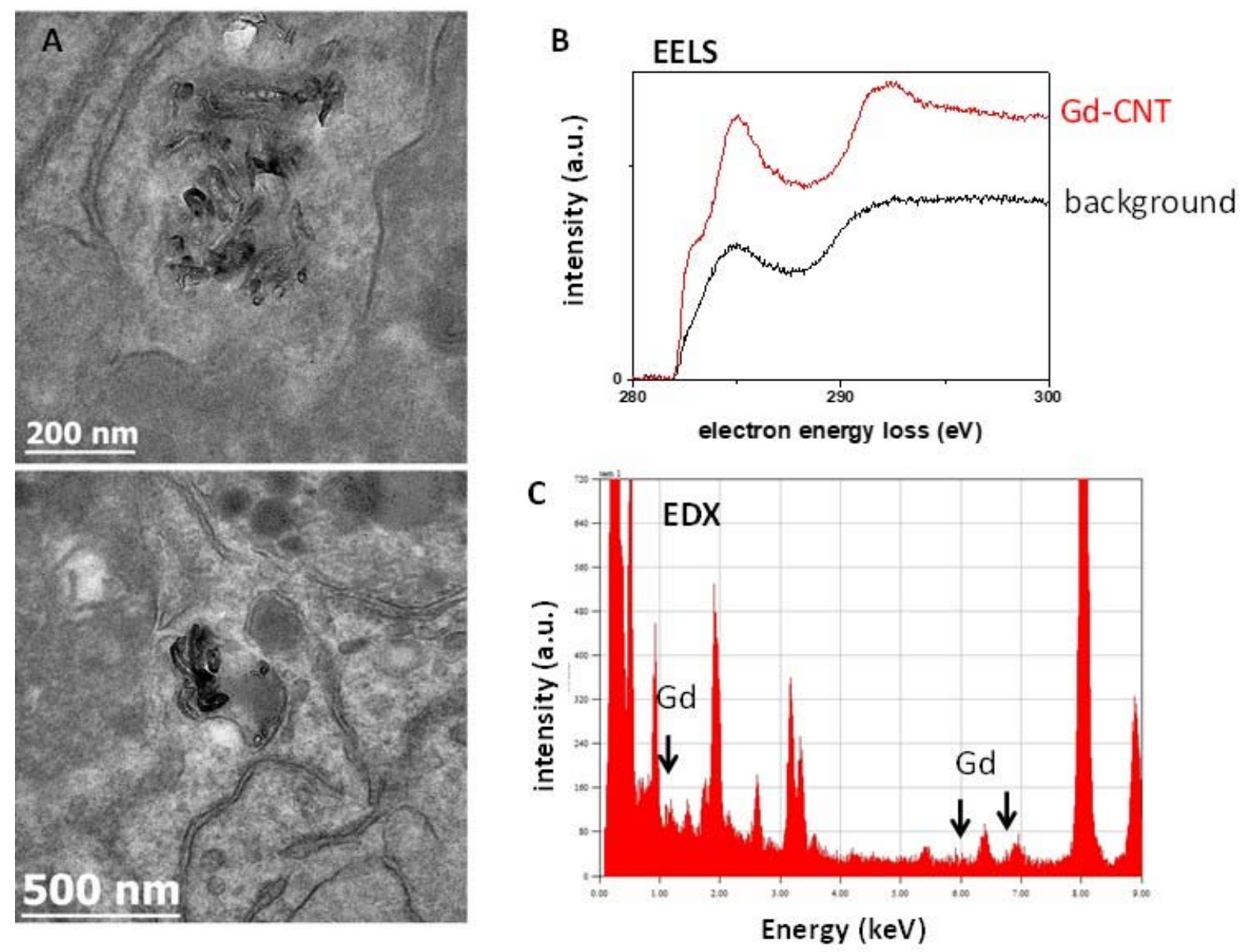

Figure 9. A) TEM of liver 6 hours after injection of Gd-CNTs. B) EELS analysis on carbon Kedge can differentiate between Gd-CNTs and the carbon rich cellular background. The strength of the first sharp peak $(285 \mathrm{eV})$ due to the $1 \mathrm{~s}$ to $2 \pi^{*}$ can be related to the predominance of $\mathrm{sp}^{2}$ hybridizations. Therefore, the strong initial peak unambiguously reveals the presence of carbon nanotubes in liver. This characteristic signal is easily differentiated from the carbon rich cellular background which shows an amorphous carbon-like K-edge. C) EDX spectrum confirms the presence of Gd on CNTs internalized by liver Kupffer cells.

Unambiguous identification of carbon nanotubes in the intracellular compartments of Kupffer cells in liver was provided by electron energy loss spectroscopy spectrum (EELS). This technique can differentiate graphitic $\mathrm{sp}^{2}$ signal from CNTs and amorphous carbon signal either 
from the endogenous cellular background or from carbon membranes present on TEM grids (Figure 9).$^{[41]}$ Moreover, EDX spectroscopy also confirmed the presence of gadolinium colocalizing with carbon nanotubes within membrane-bound intracellular compartments that are presumably lysosomes, in both liver and spleen (Figure 9). Thus, the increased MR signal in these organs is unambiguously related to the rapid uptake of CNTs by the reticuloendothelial system, as confirmed by histology and TEM. The massive and transient signal enhancement in bladder also suggests that a significant fraction of Gd-CNTs is eliminated by renal clearance. Both histology and TEM confirmed MRI results showing that Gd-CNTs do not accumulate in the kidneys (as only one isolated aggregate was observed in histological sections), which is consistent with effective elimination in the urine (Supporting Figure S7). Overall, our findings are consistent with the recent SPECT/CT imaging study on functionalized CNTs radiolabeled with ${ }^{111}$ Indium-DTPA. Different distribution profiles have been reported with a balance of renal clearance versus RES accumulation depending on the degree of chemical functionalization of the nanotubes. ${ }^{[16 a]}$ Individual functionalized CNTs with outer diameter lower than the glomerular filtration cut-off have been shown to escape the glomerular kidney filter to be excreted in urines in several studies. ${ }^{[42]}$ In contrast, most of the largest CNTs or CNTs forming bundles in vivo are uptaken in liver, spleen and lung. ${ }^{[5]}$ Therefore, full dispersion (or individualization) of CNTs which is favored by chemical functionalization appears to be a pivotal factor determining their excretion profile. In the present study, a single bolus injection of a relatively high dose of Gd-CNTs $(250 \mu \mathrm{g}$ versus $50 \mu \mathrm{g}$ and $10 \mu \mathrm{g}$ in two recent studies $^{[16 a, 26]}$ ) was used with no acute or sub-acute effect on the animal behavior apparent (until day 8 post-injection) and with no signs of tissue damage, inflammatory response and cellular toxicity that could be noticed on histological and TEM micrographs in liver, spleen, kidney and lung. This clearly indicates that the dispersion of the functionalized nanotubes in physiological saline medium at the concentration of $5 \mathrm{mg} / \mathrm{mL}$ was appropriate for intravenous administration. Moreover, unlike previous studies reporting accumulation of CNTs in the lungs after their 
systemic administration, ${ }^{[5]}$ the lung histological specimens show very few nanotubes despite the high dose injected (Supporting Figure S7). The lack of lung accumulation is a further indication of the good dispersibility of Gd-CNTs which persists in blood circulation. The ultrastructural examination of Gd-CNTs in liver and spleen also revealed that well individualized CNTs could be sequestered into intracellular compartment while keeping their native hollow and elongated structure (Figure 8). A similar intracellular distribution of CNTs was still observed 8 days after injection. This suggests that individual as well as CNT clusters were internalized in the first hours post-injection.

\section{Conclusion}

In conclusion, we covalently functionalized oxidized MWCNTs with DTPA for chelation of $\mathrm{Gd}^{3+}$ and the Gd-CNTs were administrated intravenously to mice. The CNT uptake in liver and spleen could be successfully assessed using in vivo MRI owing to the preserved capacity of GdCNTs to generate signal enhancement after cellular internalization. In addition, the rapid clearance into the urines of extracellular functionalized CNTs confirmed previous studies using other different imaging modalities. ${ }^{[43]}$ Overall, this study represents the first success in monitoring the CNT distribution with functionalized tubes used to generate a $\mathrm{T}_{1}$ positive $\mathrm{MR}$ contrast after intravenous injection. This work shows that combining the properties of $\mathrm{Gd}^{3+}$ and CNTs allows performing MRI in vivo. MRI has a high resolution compared to other imaging techniques and can thus be used as an alternative imaging method to track CNTs. Despite the fact that the amount of gadolinium chelates injected with nanotubes is far lower the gadolinium dose in clinical practice $(5.75 \mu \mathrm{mol} / \mathrm{kg}$ versus $100 \mu \mathrm{mol} / \mathrm{kg}$ body weight), the high longitudinal relaxivity of Gd-CNT conjugates enabled their detection with $\mathrm{T}_{1}$-weighting in mice using a 4.7 T scanner. In contrast to other strategy using nanoparticles to enhance MR contrast of carbon materials, the present approach exploits paramagnetic molecular chelates that are widely used in the clinics and minimally interfere with the structure, dispersability, functionalization, loading capacity and degradability of CNTs. The approach to material design described here 
could open new routes to highly dispersible nanomaterials functionalizable with significant payloads for drug delivery, tumor targeting and photothermal therapy or vaccination. The second aspect is the retention of $\mathrm{T}_{1}$-weighting properties for MRI following cellular uptake, which may provide possibilities for study the biodistribution of functionalized CNTs and for image guided delivery.

\section{Experimental Section}

Preparation of MWCNTs

MWCNTs (20-30 nm diameter, 0.5-2 $\mu \mathrm{m}$ length, 95\% purity; batch $1240 \mathrm{XH})$ were purchased from Nanostructured and Amorphous Materials. When stated, suspensions of CNTs were sonicated in a water bath (Transsonics Digitals Elma, $20 \mathrm{~W}, 40 \mathrm{kHz}$ ). All reagents and solvents were purchased from different commercial suppliers and used as received. In particular, xylenol orange tetrasodium salt was purchased from Alfa Aesar. Thermogravimetric analyses were performed by using a TGA Q500 TA instrument with a ramp of $10{ }^{\circ} \mathrm{C} \cdot \mathrm{min}^{-1}$ under $\mathrm{N}_{2}$ using a flow rate of $60 \mathrm{~mL} \cdot \mathrm{min}^{-1} .{ }^{1} \mathrm{H}$ and ${ }^{13} \mathrm{C}$ NMR spectra were recorded on Bruker DPX 300 instrument. The peak values were obtained as ppm $(\delta)$ and referenced to the solvent. The resonance multiplicity is indicated as s (singlet), br s (broad singlet), and m (multiplet). LC/MS analyses were performed on ThermoFisher Finnigan 6 LCQ Advantage Max. TLC was performed on aluminum sheets coated with silica gel 60 F254 (Merck, Darmstadt). Chromatographic purification was done with Merck silica gel (Kiesegel 60, 40-60 $\mu \mathrm{m}, 230-400$ mesh ASTM). Dialysis was performed using Spectra/Por ${ }^{\circledR}$ dialysis membrane, MWCO: 1214,000 Da.

Synthesis of DTPA ligand 2

\section{[(2-Bromo-ethyl)-tert-butoxycarbonylmethyl-amino]-acetic acid tert-butyl ester 9}

To a solution of tert-butylbromoacetate $(9.96 \mathrm{~mL}, 0.067 \mathrm{~mol})$ and $N, N$-diisopropylethylamine (DIEA) (19.5 mL, $0.11 \mathrm{~mol})$ in DMF $(25 \mathrm{~mL})$ was added dropwise a solution of 2- 
bromoethylamine hydrobromide $(4.61 \mathrm{~g}, 0.022 \mathrm{~mol})$ in DMF $(20 \mathrm{~mL})$. The reaction mixture was stirred at room temperature for 1 day. Water and ethyl acetate were added and the two phases were separated. The organic phase was washed with water (3 times) and brine, dried over $\mathrm{Na}_{2} \mathrm{SO}_{4}$, filtered and evaporated under vacuum. The crude was purified by chromatography on silica gel using ethyl acetate/cyclohexane from 3:97 to 7/93 as eluant. 3.73 g of compound 9 was obtained as colorless oil (yield: 47\%). The ${ }^{1} \mathrm{H}$ NMR spectrum was in agreement with literature. ${ }^{[4]}$

\section{6-Benzyloxycarbonylamino-2-\{bis-[2-(bis-tert-butoxycarbonylmethyl-amino)-ethyl]- amino\}-hexanoic acid tert-butyl ester 10}

To a solution of the brominated compound 9 (6.54 g, $0.019 \mathrm{~mol})$ and DIEA $(3.87 \mathrm{~mL}, 0.022$ mol) in acetonitrile $(32 \mathrm{~mL})$ was added a solution of $\mathrm{H}-\mathrm{Lys}(\mathrm{Z})-\mathrm{OtBu} \cdot \mathrm{HCl}(2.78 \mathrm{~g}, 0.0074 \mathrm{~mol})$ in a phosphate buffer $\left(\mathrm{Na}_{2} \mathrm{HPO}_{4}: 1.1 \mathrm{~g} / \mathrm{KH}_{2} \mathrm{PO}_{4}: 0.137 \mathrm{~g}, \mathrm{pH} 8\right)(36 \mathrm{~mL})$. The reaction mixture was vigorously stirred at $40{ }^{\circ} \mathrm{C}$ for 3 days. Water and ethyl acetate were added and the two phases were separated. The organic phase was washed with water (twice) and brine, dried over $\mathrm{Na}_{2} \mathrm{SO}_{4}$, filtered and evaporated under vacuum. The crude was purified by chromatography on silica gel using ethyl acetate/cyclohexane from from 20/80 to 60/40 as eluant. $3.53 \mathrm{~g}$ of compound 10 was obtained as yellow oil (yield: 54\%). The ${ }^{1} \mathrm{H}$ NMR spectra were in agreement with literature. ${ }^{[28]}$ LC-MS (ESI): m/z 879.6 [M+ H] $]^{+}$.

6-Amino-2-\{bis-[2-(bis-tert-butoxycarbonylmethyl-amino)-ethyl]-amino\}-hexanoic acid tert-butyl ester 2

To a solution of compound $10(1.77 \mathrm{~g}, 2.0 \mathrm{mmol})$ in methanol $(20 \mathrm{~mL})$ was added a catalytic amount of $\mathrm{Pd} / \mathrm{C}$. The mixture was stirred at room temperature under a hydrogen atmosphere for $17 \mathrm{~h}$. The suspension was filtered over celite and the filtrate was evaporated under vacuum to give compound 2 as colorless oil $(1.33 \mathrm{~g}, 85 \%)$ with a satisfactory purity. The ${ }^{1} \mathrm{H}$ and ${ }^{13} \mathrm{C}$ NMR spectrum was in agreement with literature (Anelli, Bioconjugate Chem. 1999, 137). LC-MS (ESI): $m / z 745.5[\mathrm{M}+\mathrm{H}]^{+}$. 


\section{Synthesis of Gd-DTPA ligand 8}

\section{6-Acetylamino-2-\{[2-(bis-tert-butoxycarbonylmethyl-amino)-ethyl]-[2-(tert-}

butoxycarbonylmethyl-isopropoxycarbonylmethyl-amino)-ethyl]-amino\}-hexanoic acid tert-butyl ester 6

To a solution of compound $2(166 \mathrm{mg}, 0.23 \mathrm{mmol})$ in methanol $(2.3 \mathrm{~mL})$ was added acetic anhydride $(24 \mu \mathrm{L}, 0.25 \mathrm{mmol})$, and the mixture was stirred at room temperature. The reaction was monitored by TLC. After $7 \mathrm{~h}$, the reaction mixture was evaporated under vacuum to give $171 \mathrm{mg}$ of compound 6 as a yellow oil (yield: 98\%) with a satisfactory purity. ${ }^{1} \mathrm{H}$ NMR (300 $\mathrm{MHz}, \mathrm{CDCl}_{3}$ ): $\delta 6.05$ (br s, $\left.1 \mathrm{H}\right), 3.41(\mathrm{~s}, 8 \mathrm{H}), 3.31-3.16(\mathrm{~m}, 3 \mathrm{H}), 2.84-2.58(\mathrm{~m}, 8 \mathrm{H}), 1.91$ (s, $3 \mathrm{H}), 1.64-1.22(\mathrm{~m}, 51 \mathrm{H}) .{ }^{13} \mathrm{C} \mathrm{NMR}\left(75 \mathrm{MHz}, \mathrm{CDCl}_{3}\right): 172.9,170.8,170.2,81.0,80.8,63.9$, 56.0, 53.7, 50.2, 39.4, 29.5, 29.0, 28.4, 28.3, 23.6, 23.4. LC/MS (ESI): $m / z 787.3[\mathrm{M}+\mathrm{H}]^{+}$.

\section{6-Acetylamino-2-\{bis-[2-(bis-carboxymethyl-amino)-ethyl]-amino\}-hexanoic acid 7}

A solution of compound $6(39 \mathrm{mg}, 0.050 \mathrm{mmol})$ in TFA $(0.9 \mathrm{~mL})$ and water $(0.1 \mathrm{~mL})$ was stirred at room temperature for 1 day. The reaction was monitored by LC/MS. The reaction mixture was precipitated in diethyl ether and filtered. The solid was dissolved in water and the solution was lyophilized to give $17 \mathrm{mg}$ of compound 7 as a beige solid (yield: $68 \%$ ) with a satisfactory purity. ${ }^{1} \mathrm{H}$ NMR (300 MHz, DMSO): $\delta 3.35$ (s, $\left.8 \mathrm{H}\right), 3.41-3.28$ (m, $\left.3 \mathrm{H}\right), 3.10-2.98$ (m, $8 \mathrm{H}), 1.90-1.81(\mathrm{~m}, 2 \mathrm{H}), 1.79$ (s, $3 \mathrm{H}), 1.52-1.31$ (m, $4 \mathrm{H}) .{ }^{13} \mathrm{C}$ NMR (75 MHz, DMSO): 172.6, 171.4, 169.1, 63.9, 54.4, 50.6, 50.3, 38.3, 29.1, 27.1, 23.6, 22.6. LC/MS (ESI): m/z 507.3 $[\mathrm{M}+\mathrm{H}]^{+}$

\section{Gd-DTPA ligand 8}

A solution of DTPA ligand 7 (9.4 mg) in a solution of gadolinium(III) perchlorate aqueous solution (Strem Chemicals) $(14.9 \mu \mathrm{mol})$ in water $(9.4 \mathrm{~mL})$ was stirred at room temperature for 3 days. An aliquot of the solution was titrated with xylenol orange to check that there was no more free Gd. The solution was lyophilized to obtain Gd-DTPA ligand 8. 


\section{ox-CNTs 1}

A suspension of pristine MWCNTs $(1 \mathrm{~g})$ in a mixture of sulfuric acid 98\% (108 $\mathrm{mL})$ and nitric acid $65 \%(36 \mathrm{~mL})$ was sonicated in a water bath for $24 \mathrm{~h}$. The temperature was kept below $35{ }^{\circ} \mathrm{C}$. Deionized water was then carefully added and the CNTs were filtered (Omnipore ${ }^{\mathrm{TM}}$ membrane filtration, $0.45 \mu \mathrm{m})$, re-suspended in water, filtered again until $\mathrm{pH}$ became neutral and dried under vacuum.

\section{Functionalized CNTs 3}

A suspension of ox-CNTs $1(21 \mathrm{mg})$ in oxalyl chloride $(8.5 \mathrm{~mL})$ was sonicated in a water bath for $30 \mathrm{~min}$ and then heated at reflux for $24 \mathrm{~h}$. After evaporation under vacuum, the acyl chlorideCNTs were dispersed in a solution of DTPA ligand $2(237 \mathrm{mg})$ in dry THF $(10 \mathrm{~mL})$. The mixture was sonicated for $2 \mathrm{~min}$ and then heated at reflux for $60 \mathrm{~h}$. The suspension was filtered over a PTFE membrane $\left(0.1 \mu \mathrm{m}\right.$, Omnipore ${ }^{\mathrm{TM}}$, Millipore). The solid recovered on the filter was dispersed in DMF (100 mL), sonicated for $3 \mathrm{~min}$ in a water bath, and filtered over a PTFE membrane $(0.1 \mu \mathrm{m})$. This sequence was repeated with DMF, methanol (twice), and dichloromethane. The resulting solid was dried under vacuum.

\section{DTPA/CNTs 4}

A suspension of the functionalized CNTs $3(21 \mathrm{mg})$ in TFA $(1.8 \mathrm{~mL})$ and water $(200 \mu \mathrm{L})$ was sonicated in a water bath for $3 \mathrm{~min}$ and stirred at room temperature for $2 \mathrm{~h}$. A solution of TFA $(1.8 \mathrm{~mL})$ and water $(200 \mu \mathrm{L})$ was added and the mixture was further stirred for $18 \mathrm{~h}$. After evaporation under vacuum, diethyl ether was added and the mixture was evaporated under vacuum. The residue was dispersed in water and dialyzed against water for 2 days. The suspension was lyophilized to obtain DTPA/CNTs 4.

\section{Gd-CNTs 5}

A suspension of DTPA/CNTs $4(8.3 \mathrm{mg})$ in a solution of gadolinium(III) perchlorate $50 \%$ aqueous solution $(4.04 \mu \mathrm{mol})$ in water $(8.3 \mathrm{~mL})$ was sonicated for $30 \mathrm{~min}$ and stirred at $\mathrm{rt}$ for $24 \mathrm{~h}$. An aliquot of the suspension was centrifuged. The supernatant was titrated with xylenol 
orange to assess the amount of free Gd. The reaction mixture was then dialyzed against water for 2 days. The suspension was lyophilized to obtain Gd-CNTs 5.

Aberration-corrected high resolution transmission electron microscopy (HR-TEM), energydispersive $X$-ray spectroscopy (EDX) and high angle annular dark field scanning transmission electron microscopy (HAADF)-STEM. HR-TEM investigations were performed with the newly developed JEOL ARM $200 \mathrm{~F}$ microscope operating at $80 \mathrm{kV}$. This microscope is equipped together with a CEOS aberration corrector, a cold field emission gun, a Gatan GIF quantum ER and a JEOL EDX Diode. ${ }^{[45]}$

HAADF-STEM images and EDX spectra were acquired on a FEI Tecnai G2 F20 HRTEM operated at $200 \mathrm{kV}$ and equipped with an EDAX super ultra-thin window (SUTW) X-ray detector. Samples were deposited on lacey carbon $\mathrm{Cu}$ TEM grids (Agar).

Inductively coupled plasma atomic emission spectrometry (ICP-AES) analysis. Determination of gadolinium, iron and nickel content in CNTs was performed by ICP-AES analysis (Agilent $7500 \mathrm{ce})$. The samples were digested in concentrated $\operatorname{HNO} 3(3: 1, \mathrm{v} / \mathrm{v})$ solution and diluted with ultrapure water for the analysis.

Magnetization measurements. Magnetic measurements were carried out on a Quantum Design MPMS-5S SQUID Magnetometer. Field-dependent magnetization curves were measured at 5K as function of the external field in the range $0-5$ Tesla. Field-cooled (FC) and Zero-fieldcooled (ZFC) temperature-dependant magnetization was measured using a magnetic field of 100 Oe or 1000 Oe and the sample was cooled with the same magnetic field.

NMRD and relaxivity measurements. The frequency dependence of the ${ }^{1} \mathrm{H}$ relaxation for the aqueous nanotube suspensions was recorded over the frequency range $0.01-20 \mathrm{MHz}$ using a Spinmaster FFC-2000 Fast Field Cycling NMR Relaxometer (Stelar SRL, Mede, Italy). T1 measurements were performed as a function of external field, $\mathrm{B}_{0}$, with standard pulse sequences incorporating $\mathrm{B}_{0}$ field excursions. The temperature of the samples was maintained at $25 \pm 1{ }^{\circ} \mathrm{C}$ 
using a thermostated airflow system. All of the ${ }^{1} \mathrm{H}$ magnetisation recovery curves were singly exponential within experimental error and the random errors in fitting $\mathrm{T}_{1}$ were always less than 1\%. T2 was measured using the CPMG pulse sequence at 20, 40 and $60 \mathrm{MHz}$ using the Stelar spectrometer and a reconditioned Bruker WP80 electromagnet. $\mathrm{T}_{2}$ was measured using the CPMG pulse sequence at 20, 40 and $60 \mathrm{MHz}$. Images of CNT liquid suspensions, agarose gel $(0.3 \%)$ and cell samples $\left(7 \times 10^{6}\right.$ cells in $70 \mu \mathrm{L}$ agarose $)$ were obtained on a Bruker Bio-Spec 47/40 USR scanner operating at 4.7T $(200 \mathrm{MHz})$ using fast spin echo sequences with variable echo time $T_{E}$ and repetition time $T_{R} . T_{1}$ and $T_{2}$ were deduced from monoexponential fit of $M R$ signal as function of $T_{R}$ and $T_{E}$, respectively, using the ParaVision software.

Cell culture, cell labeling, cell metabolic activity and cell preparation for TEM

The murine macrophage RAW 264.7 cells were maintained as monolayer cultures in RPMI (Roswell Park Memorial Institute) medium supplemented with 10\% fetal bovine serum, 1\% penicillin-streptomycin at $37^{\circ} \mathrm{C}$ and $5 \%$ of $\mathrm{CO}_{2}$. For cell labeling, cells were incubated with complete RPMI medium supplemented with CNTs at concentration of $10,20,50,100 \mu \mathrm{g} / \mathrm{mL}$ for 20 hours at $37^{\circ} \mathrm{C}$.

Cell metabolic activity was assessed by the Alamar blue test in 48 -well plates seeded with cells the day before labeling. The labeled macrophages were washed twice in PBS and were incubated with 10\% Alamar Blue in culture medium for 2 hours. The fluorescence in cell medium due to the reduction of resazurin (oxidized form) to resorufin by cell activity was quantified on a FLUOstar OPTIMA microplate reader (excitation $550 \mathrm{~nm}$, emission $590 \mathrm{~nm}$ ) and normalized to the fluorescence signal of the control non-labeled cells. The conditions were run in quadruplicate.

For TEM observation, cells were trypsinized, centrifuged at $1200 \mathrm{rpm}$ for $5 \mathrm{~min}$, washed in 0.1 $\mathrm{M}$ cacodylate buffer and fixed with $2.5 \%$ glutaraldehyde in cacodylate buffer at $4{ }^{\circ} \mathrm{C}$ for 30 min. Cells were then post-fixed with osmium tetraoxide $1 \%$ and passed through uranyl acetate. Samples were then dehydrated in an ethanol series (30\%-100\%) and embedded in epoxy 
medium (EPON 812; Shell Chemical, San Francisco, California). Thin sections (70 nm) were collected onto 200 mesh cooper grids, and counterstained with lead citrate before examination with Zeiss EM902 electron microscope operated at $80 \mathrm{kV}$ (MIMA2- UR1196 Génomique et Physiologie de la Lactation, INRA, Plateau de Microscopie Electronique 78352, Jouy en Josas, France).

Imaging flow cytometry. For imaging flow cytometry analysis, labeled macrophages were trypsinized, fixed with $4 \%$ formaldehyde in PBS and centrifuged to obtain a pellet of about $10^{6}$ cells in $50 \mu \mathrm{L}$. Cell images were acquired using the ImageStream ${ }^{\mathrm{X}}$ multispectral imaging flow cytometer (Amnis Corporation), collecting 10,000 events per sample at 40x magnification. Dark field images were acquired using a $785 \mathrm{~nm}$ laser. Cell images were analyzed using IDEAS ${ }^{\circledR}$ image-analysis software (Amnis). Gating on bivariate plot of aspect ratio versus cell area was first used to isolate a population of single cells. Cells within the focal plane were further selected using a two-dimensional plot of image contrast versus root mean squared (RMS) gradient. Mean pixel feature on bright field images versus intensity feature on dark field images was used as a biparametric dot plot to discriminate cells with respect to their CNT uptake.

Animal experiments and MRI protocol. Animal experiments were conducted in line with French Agriculture Ministry guidelines. During the MRI protocol the animals were anesthetized with 2\% isofluorane (Aerrane, Baxter, Maurepas, France) supplied in an air mixture, while their body temperature was kept constant by circulating thermostated warm water. Twelve eightweeks-old female C57/B16 mice (mean weight 20.5 \pm 1 g) (Janvier, France) were used in this study.

The mice were imaged by the whole body MRI 20 min before injection and 10, 75, 120, 320 min after injection of Gd-CNTs in the retro-orbital sinus at a dose of 1 or $5 \mathrm{mg} / \mathrm{mL}$ suspended in a $100 \mu \mathrm{L}$ physiological saline medium. Three animals were analyzed at each time point. MRI was performed on a Biospec 47/40 USR (40-cm bore actively shielded 4.7 Tesla magnet) 
scanner interfaced to ParaVision software (both provided from Bruker Biospin GmbH, Rheinstetten, Germany). The whole body imaging protocol was performed with a volume transmission/reception RF coil for mice (Bruker), using a fast spin echo (Turbo RARE) sequence with a $T_{E}$ of $12 \mathrm{~ms}$ and a $T_{R}$ of $445 \mathrm{~ms}$ ( $\mathrm{T}_{1}$-weighted sequence), flip angle of $180^{\circ}, 30$ averages, in-plane resolution of $150 \mu \mathrm{m}$, slice thickness of $500 \mathrm{~nm}$, for a total of seven slices. At the end of the experimental protocol, mice were sacrificed with a lethal injection of sodium pentobarbital. Livers, spleens, lungs and kidneys were excised and prepared for histological and TEM characterizations.

Image processing and analysis were made with the open source software OsiriX (3.9.2. version). The percentage of contrast enhancement was defined as $\frac{\left(C N R_{\text {post-injection }}-C N R_{\text {pre-injection }}\right)}{C N R_{\text {pre-injection }}} \times 100$, with the contrast-to-noise ratio $(\mathrm{CNR})$ measured in different organs of interest at the different investigation time points. CNR was defined as $\frac{\left(S N R_{R O I}-S N R_{\text {ref }}\right)}{S N R_{\text {ref }}}$ with $S N R=\frac{\text { Mean }_{\text {Signal }}}{S D_{\text {Noise }}}$, where ref denotes the water tube reference and SD is the standard deviation of the noise in the image. A water tube, used as a reference, was positioned near the mice to enable CNR measurement and ${ }^{1} \mathrm{H}$ NMR signal normalization. ROIs encompassing the liver parenchyma, the whole spleen, the kidneys and the bladder were manually selected for signal measurement.

Histology. After excision, pieces of liver, spleen, lung and kidney were fixed with $\mathrm{pH} 7.4$ phosphate-buffered $10 \%$ formalin and processed by embedding in paraffin. Sections, $\sim 6 \mu \mathrm{m}$ thick, were evaluated after hematoxylin and eosin or Nuclear Fast Red and Pearl's staining (all from Sigma-Aldrich, Steinheim, Germany).

Transmission electron microscopy in organs. Organs were cut into $1 \mathrm{~mm}^{3}$ pieces after excision and fixed with $2 \%$ glutaraldehyde in $0.1 \mathrm{M}$ sodium cacodylate buffer, postfixed with $1 \%$ osmium tetroxide containing $1.5 \%$ potassium cyanoferrate, gradually dehydrated in ethanol and 
embedded in Epon. Thin sections $(70 \mathrm{~nm})$ of selected zones were observed with Zeiss EM902 electron microscope operated at $80 \mathrm{kV}$ as described above for cells.

Ultra-thin sections (30 nm) of organs were specially prepared for high resolution imaging, EDX analysis and Electron Energy Loss Spectroscopy (EELS) analysis on the carbon K-edge at 284 $\mathrm{eV}$, performed on the aberration-corrected JEOL ARM 200F operating at $80 \mathrm{kV}$.

Acknowledgment I.M. and C. M.-M. contributed equially to this work. The authors thank C. Péchoux and S. Chat for electron microscopy, C. Fabbro for TGA measurements, O. Clément and L. Pidial for the access to the Small Animal Imaging Platform Paris-Descartes PARCCHEGP and to the animal facility, N. Bogetto and the platform Imagoseine (University Paris Diderot, Institut Jacques Monod) for imaging flow cytometry. This work was supported by ANR P2N (NANOTHER project 2010-NANO-008-398 04) and by the Region Ile-de-France (convention SESAME E1845 for the JEOL ARM 200F electron microscope recently installed at the Paris Diderot University). EP, BB, CMM, and AB acknowledge the EU FP7-ITN MarieCurie Network programme RADDEL (290023). EP is enrolled in the UAB PhD program.

Supporting Information. Supporting Information is available from the Wiley Online Library or from the author.

TEM of ox- and Gd-CNTs, HAADF-STEM images of ox-CNTs, synthesis of DTPA ligand 2, HAADF-STEM images and EDX spectra of Gd-CNTs and DTPA/CNTs, synthesis of GdDTPA/ligand 8, cell viability, histology in liver, spleen, kidney and lung, and TEM, EELS and EDX in spleen.

\section{References}

[1] a) K. Kostarelos, A. Bianco, M. Prato, Nat Nano 2009, 4, 627; b) B. S. Wong, S. L. Yoong, A. Jagusiak, T. Panczyk, H. K. Ho, W. H. Ang, G. Pastorin, Advanced Drug Delivery Reviews 2013, 65, 1964.

[2] a) S. Lanone, P. Andujar, A. Kermanizadeh, J. Boczkowski, Advanced Drug Delivery Reviews 2013, 65, 2063; b) J. Kolosnjaj-Tabi, H. Szwarc, H. Moussa, in The Delivery of Nanoparticles, (Ed: D. A. A. H. (Ed.)), InTech, 2012.

[3] A. Bianco, K. Kostarelos, M. Prato, Chemical Communications 2011, 47, 10182.

[4] A. Battigelli, C. Ménard-Moyon, T. Da Ros, M. Prato, A. Bianco, Advanced Drug Delivery Reviews 2013, 65, 1899.

[5] H. Ali-Boucetta, K. Kostarelos, Advanced Drug Delivery Reviews 2013, 65, 2111. 
[6] a) H. Dumortier, S. Lacotte, G. Pastorin, R. Marega, W. Wu, D. Bonifazi, J.-P. Briand, M. Prato, S. Muller, A. Bianco, Nano Letters 2006, 6, 1522; b) K. Kostarelos, L. Lacerda, G. Pastorin, W. Wu, WieckowskiSebastien, J. Luangsivilay, S. Godefroy, D. Pantarotto, J.-P. Briand, S. Muller, M. Prato, A. Bianco, Nat Nano 2007, 2, 108.

[7] a) C. Fabbro, H. Ali-Boucetta, T. D. Ros, K. Kostarelos, A. Bianco, M. Prato, Chemical Communications 2012, 48, 3911; b) B. R. Smith, C. Zavaleta, J. Rosenberg, R. Tong, J. Ramunas, Z. Liu, H. Dai, S. S. Gambhir, Nano Today 2013, 8, 126.

[8] R. Singh, S. V. Torti, Advanced Drug Delivery Reviews 2013, 65, 2045.

[9] a) K. T. Al-Jamal, F. M. Toma, A. Yilmazer, H. Ali-Boucetta, A. Nunes, M.-A. Herrero, B. Tian, A. Eddaoudi, W. T. Al-Jamal, A. Bianco, M. Prato, K. Kostarelos, The FASEB Journal 2010, 24, 4354; b) K. Bates, K. Kostarelos, Advanced Drug Delivery Reviews 2013, 65, 2023.

[10] a) S. H. Ku, M. Lee, C. B. Park, Advanced Healthcare Materials 2013, 2, 244; b) T. R. Nayak, L. Jian, L. C. Phua, H. K. Ho, Y. Ren, G. Pastorin, ACS Nano 2010, 4, 7717.

[11] a) L. Tong, Y. Liu, B. D. Dolash, Y. Jung, M. N. Slipchenko, D. E. Bergstrom, J.-X. Cheng, Nat Nano 2012, 7, 56; b) I. Marangon, N. Boggetto, C. Ménard-Moyon, E. Venturelli, M.-L. Béoutis, C. Péchoux, N. Luciani, C. Wilhelm, A. Bianco, F. Gazeau, Nano Letters 2012, 12, 4830.

[12] a) L. G. Delogu, G. Vidili, E. Venturelli, C. Ménard-Moyon, M. A. Zoroddu, G. Pilo, P. Nicolussi, C. Ligios, D. Bedognetti, F. Sgarrella, R. Manetti, A. Bianco, Proceedings of the National Academy of Sciences 2012, 109, 16612; b) A. De La Zerda, C. Zavaleta, S. Keren, S. Vaithilingam, S. Bodapati, Z. Liu, J. Levi, B. R. Smith, T.-J. Ma, O. Oralkan, Z. Cheng, X. Chen, H. Dai, B. T. Khuri-Yakub, S. S. Gambhir, Nat Nano 2008, 3, 557.

[13] C. Zavaleta, A. de la Zerda, Z. Liu, S. Keren, Z. Cheng, M. Schipper, X. Chen, H. Dai, S. S. Gambhir, Nano Letters 2008, 8, 2800.

[14] A. E. Porter, M. Gass, K. Muller, J. N. Skepper, P. A. Midgley, M. Welland, Nat Nano 2007, 2, 713 .

[15] A. A. Bhirde, V. Patel, J. Gavard, G. Zhang, A. A. Sousa, A. Masedunskas, R. D. Leapman, R. Weigert, J. S. Gutkind, J. F. Rusling, ACS Nano 2009, 3, 307.

[16] a) K. T. Al-Jamal, A. Nunes, L. Methven, H. Ali-Boucetta, S. Li, F. M. Toma, M. A. Herrero, W. T. Al-Jamal, H. M. M. ten Eikelder, J. Foster, S. Mather, M. Prato, A. Bianco, K. Kostarelos, Angewandte Chemie International Edition 2012, 51, 6389; b) J. T.-W. Wang, L. Cabana, M. Bourgognon, H. Kafa, A. Protti, K. Venner, A. M. Shah, J. K. Sosabowski, S. J. Mather, A. Roig, X. Ke, G. Van Tendeloo, R. T. M. de Rosales, G. Tobias, K. T. Al-Jamal, Advanced Functional Materials 2014, 24, 1880.

[17] H. Gong, R. Peng, Z. Liu, Advanced Drug Delivery Reviews 2013, 65, 1951.

[18] a) A. Al Faraj, K. Cieslar, G. Lacroix, S. Gaillard, E. Canet-Soulas, Y. Cremillieux, Nano Lett 2009, 9, 1023; b) B.-T. Doan, J. Seguin, M. Breton, R. L. Beherec, M. Bessodes, J. A. Rodríguez-Manzo, F. Banhart, J.-C. Beloeil, D. Scherman, C. Richard, Contrast Media \& Molecular Imaging 2012, 7, 153.

[19] V. Orazio, L. D. Suzanne, P. Andrea, C. Alfred, Nanotechnology 2011, 22, 095706.

[20] a) M. Yin, M. Wang, F. Miao, Y. Ji, Z. Tian, H. Shen, N. Jia, Carbon 2012, 50, 2162;

b) G. Lamanna, A. Garofalo, G. Popa, C. Wilhelm, S. Begin-Colin, D. Felder-Flesch, A. Bianco, F. Gazeau, C. Menard-Moyon, Nanoscale 2013, 5, 4412; c) H. Wu, G. Liu, Y. Zhuang, D. Wu, H. Zhang, H. Yang, H. Hu, S. Yang, Biomaterials 2011, 32, 4867.

[21] a) L. A. Tran, M. Hernández-Rivera, A. N. Berlin, Y. Zheng, L. Sampaio, C. Bové, M. d. G. Cabreira-Hansen, J. T. Willerson, E. C. Perin, L. J. Wilson, Biomaterials 2014, 35, 720; b) Q. Ma, M. Jebb, M. F. Tweedle, L. J. Wilson, Journal of Materials Chemistry B 2013, 1, 5791; c) A. M. Tang, J. S. Ananta, H. Zhao, B. T. Cisneros, E. Y. Lam, S. T. Wong, L. J. Wilson, K. K. Wong, Contrast Media \& Molecular Imaging 2011, 6, 93. 
[22] a) L. Lartigue, D. Alloyeau, J. Kolosnjaj-Tabi, Y. Javed, P. Guardia, A. Riedinger, C. Péchoux, T. Pellegrino, C. Wilhelm, F. Gazeau, ACS Nano 2013, 7, 3939; b) G. P. Kotchey, Y. Zhao, V. E. Kagan, A. Star, Advanced Drug Delivery Reviews 2013, 65, 1921; c) J.

Russier, C. Menard-Moyon, E. Venturelli, E. Gravel, G. Marcolongo, M. Meneghetti, E. Doris, A. Bianco, Nanoscale 2011, 3, 893; d) M. Levy, N. Luciani, D. Alloyeau, D. Elgrabli, V. Deveaux, C. Pechoux, S. Chat, G. Wang, N. Vats, F. Gendron, C. Factor, S. Lotersztajn, A. Luciani, C. Wilhelm, F. Gazeau, Biomaterials 2011, 32, 3988.

[23] a) B. Sitharaman, K. R. Kissell, K. B. Hartman, L. A. Tran, A. Baikalov, I. Rusakova, Y. Sun, H. A. Khant, S. J. Ludtke, W. Chiu, S. Laus, E. Toth, L. Helm, A. E. Merbach, L. J. Wilson, Chemical Communications 2005, 3915; b) R. Sethi, Y. Mackeyev, L. J. Wilson, Inorganica Chimica Acta 2012, 393, 165.

[24] B. Sitharaman, B. D. Jacobson, Y. Z. Wadghiri, H. Bryant, J. Frank, Journal of Applied Physics 2013, 113, 134308.

[25] C. Richard, B.-T. Doan, J.-C. Beloeil, M. Bessodes, E. Toth, D. Scherman, Nano Letters 2007, 8, 232.

[26] P. K. Avti, Y. Talukdar, M. V. Sirotkin, K. R. Shroyer, B. Sitharaman, Journal of Biomedical Materials Research Part B: Applied Biomaterials 2013, 101B, 1039.

[27] W. Wu, S. Wieckowski, G. Pastorin, M. Benincasa, C. Klumpp, J.-P. Briand, R. Gennaro, M. Prato, A. Bianco, Angewandte Chemie International Edition 2005, 44, 6358.

[28] P. L. Anelli, F. Fedeli, O. Gazzotti, L. Lattuada, G. Lux, F. Rebasti, Bioconjugate Chemistry 1998, 10, 137.

[29] A. D. Sherry, W. P. Cacheris, K.-T. Kuan, Magnetic Resonance in Medicine 1988, 8, 180.

[30] a) A. Barge, G. Cravotto, E. Gianolio, F. Fedeli, Contrast Media \& Molecular Imaging 2006, 1, 184; b) S.-W. Kang, C.-M. Park, K.-H. Cho, H.-S. Han, Bull Korean Chem Soc 1993, 14, 4.

[31] a) S. Y. Hong, G. Tobias, B. Ballesteros, F. El Oualid, J. C. Errey, K. J. Doores, A. I. Kirkland, P. D. Nellist, M. L. H. Green, B. G. Davis, Journal of the American Chemical Society 2007, 129, 10966; b) S. Y. Hong, G. Tobias, K. T. Al-Jamal, B. Ballesteros, H. AliBoucetta, S. Lozano-Perez, P. D. Nellist, R. B. Sim, C. Finucane, S. J. Mather, M. L. H. Green, K. Kostarelos, B. G. Davis, Nat Mater 2010, 9, 485; c) P. Luksirikul, B. Ballesteros, G. Tobias, M. G. Moloney, M. L. H. Green, Journal of Materials Chemistry 2011, 21, 19080. [32] P. Caravan, J. J. Ellison, T. J. McMurry, R. B. Lauffer, Chemical Reviews 1999, 99, 2293.

[33] a) S. Aime, M. Botta, M. Fasano, E. Terreno, Accounts of Chemical Research 1999, 32, 941; b) P. Caravan, Accounts of Chemical Research 2009, 42, 851.

[34] a) C. Platas-Iglesias, L. Vander Elst, W. Zhou, R. N. Muller, C. F. G. C. Geraldes, T. Maschmeyer, J. A. Peters, Chemistry - A European Journal 2002, 8, 5121; b) S. Aime, L. Frullano, S. Geninatti Crich, Angewandte Chemie 2002, 114, 1059; c) J. S. Ananta, B. Godin, R. Sethi, L. Moriggi, X. Liu, R. E. Serda, R. Krishnamurthy, R. Muthupillai, R. D. Bolskar, L. Helm, M. Ferrari, L. J. Wilson, P. Decuzzi, Nat Nano 2010, 5, 815.

[35] a) C. Billotey, C. Wilhelm, M. Devaud, J. C. Bacri, J. Bittoun, F. Gazeau, Magnetic Resonance in Medicine 2003, 49, 646; b) M. Lévy, C. Wilhelm, M. Devaud, P. Levitz, F. Gazeau, Contrast Media \& Molecular Imaging 2012, 7, 373.

[36] R. Di Corato, F. Gazeau, C. Le Visage, D. Fayol, P. Levitz, F. Lux, D. Letourneur, N. Luciani, O. Tillement, C. Wilhelm, ACS Nano 2013, 7, 7500.

[37] a) E. Terreno, S. Geninatti Crich, S. Belfiore, L. Biancone, C. Cabella, G. Esposito, A. D. Manazza, S. Aime, Magnetic Resonance in Medicine 2006, 55, 491; b) S. Geninatti-Crich, I. Szabo, D. Alberti, D. Longo, S. Aime, Contrast Media \& Molecular Imaging 2011, 6, 421. [38] G. J. Strijkers, S. Hak, M. B. Kok, C. S. Springer, K. Nicolay, Magnetic Resonance in Medicine 2009, 61, 1049. 
[39] I. Marangon, N. Boggetto, C. Ménard-Moyon, N. Luciani, C. Wilhelm, A. Bianco, F. Gazeau, J Vis Exp 2013, e50566.

[40] S. H. Koenig, R. D. I. Brown, D. Adams, D. Emerson, C. G. Harrison, Investigative Radiology 1984, 19, 76.

[41] B. Reed, M. Sarikaya, J Electron Microsc 2002, 51, S97.

[42] a) L. Lacerda, M. A. Herrero, K. Venner, A. Bianco, M. Prato, K. Kostarelos, Small 2008, 4, 1130; b) A. Ruggiero, C. H. Villa, E. Bander, D. A. Rey, M. Bergkvist, C. A. Batt, K. Manova-Todorova, W. M. Deen, D. A. Scheinberg, M. R. McDevitt, Proceedings of the National Academy of Sciences 2010, 107, 12369.

[43] a) R. Singh, D. Pantarotto, L. Lacerda, G. Pastorin, C. Klumpp, M. Prato, A. Bianco, K. Kostarelos, Proc. Natl Acad. Sci. USA 2006, 103, 3357; b) M. R. McDevitt, D.

Chattopadhyay, J. S. Jaggi, R. D. Finn, P. B. Zanzonico, C. Villa, D. Rey, J. Mendenhall, C. A. Batt, J. T. Njardarson, D. A. Scheinberg, PLoS ONE 2007, 2, e907.

[44] C. M. Micklitsch, Q. Yu, J. P. Schneider, Tetrahedron Letters 2006, 47, 6277.

[45] C. Ricolleau, J. Nelayah, T. Oikawa, Y. Kohno, N. Braidy, G. Wang, F. Hue, L.

Florea, V. Pierron Bohnes, D. Alloyeau, Microscopy 2013, 62, 283. 


\section{Supporting Information}

Covalent functionalization of multi-walled carbon nanotubes with a gadolinium chelate for efficient T1-weighted magnetic resonance imaging

Iris Marangon, Cécilia Ménard-Moyon, Jelena Kolosnjaj-Tabi, Marie Lys Béoutis, Lénaic Lartigue, Damien Alloyeau, Elzbieta Pach, Belén Ballesteros, Gwennhael Autret, Tsedev Ninjbadgar, Dermot F. Brougham, Alberto Bianco, ${ }^{*}$ Florence Gazeau* 

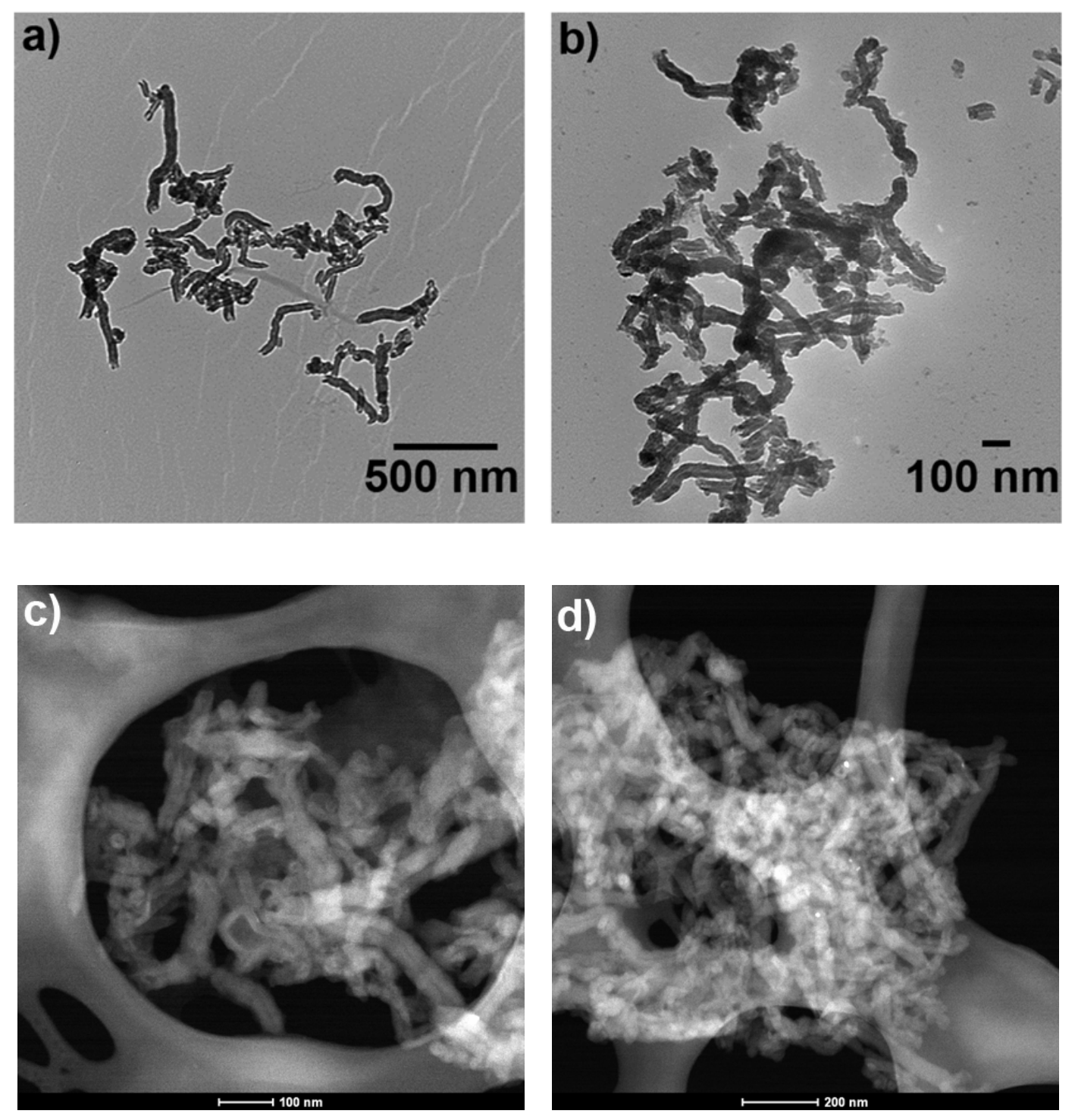

Figure S1. TEM image of ox-CNTs 1 (a) and Gd-CNTs 5 (b) (Low resolution TEM images were recorded at the RIO Microscopy Facility Platform of Esplanade Campus (Strasbourg, France)). HAADF-STEM images of ox-CNTs 1 (c, d). 


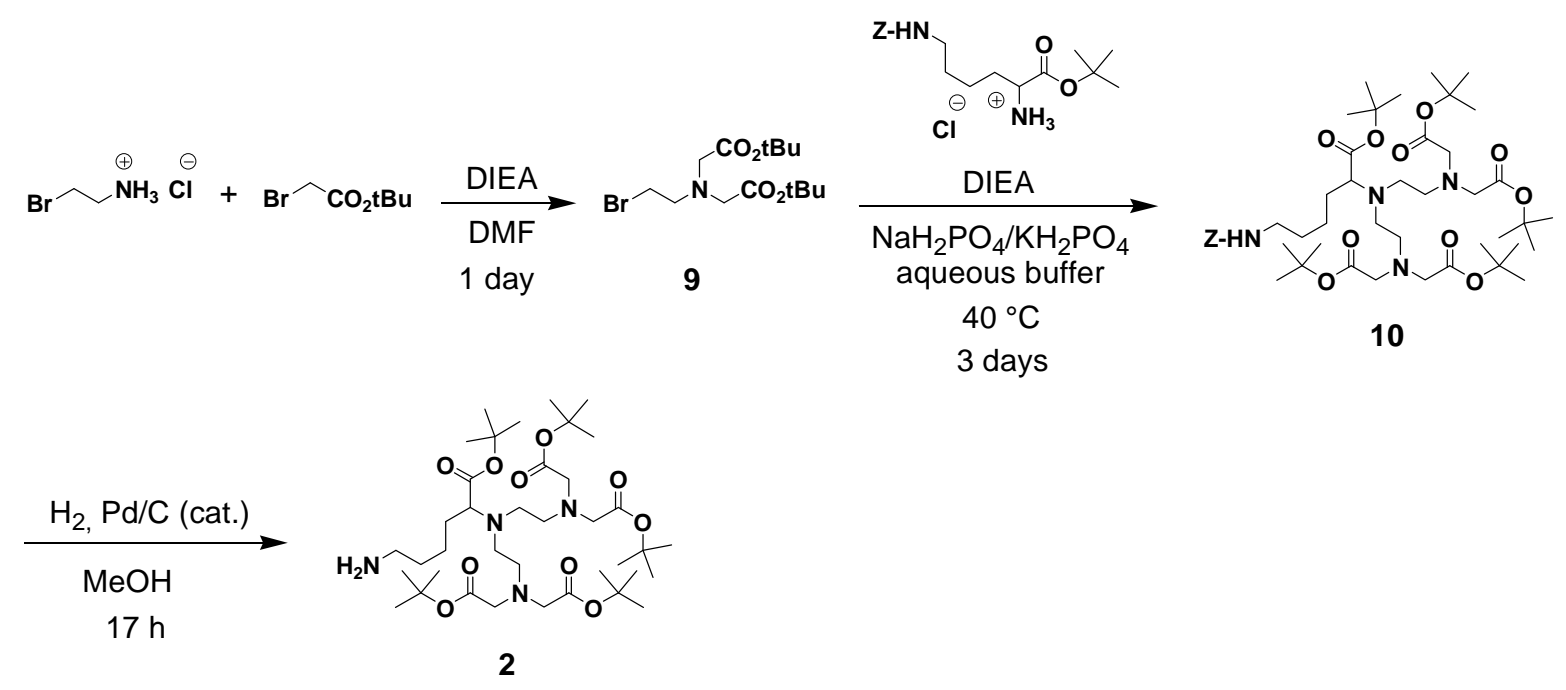

Figure S2. Synthesis of DTPA ligand 2. 

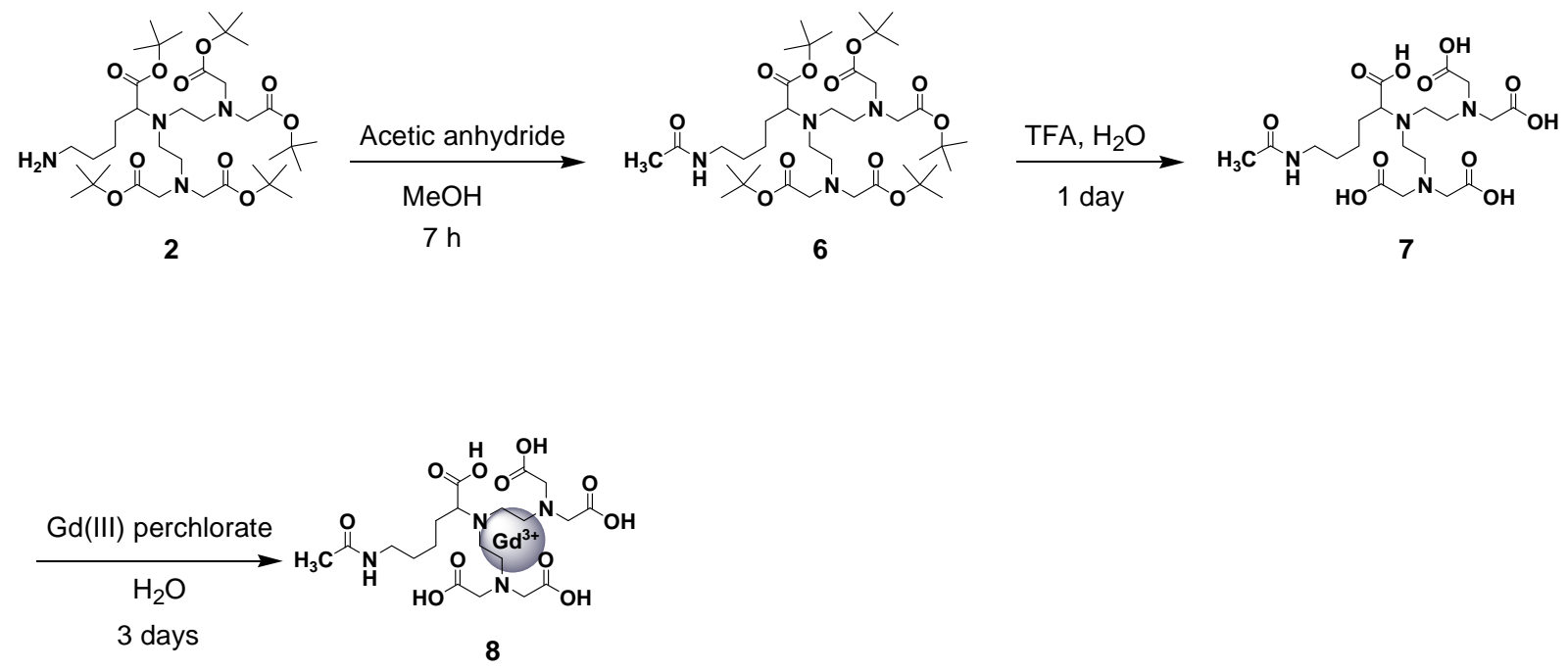

Figure S3: Synthesis of Gd-DTPA ligand 8.

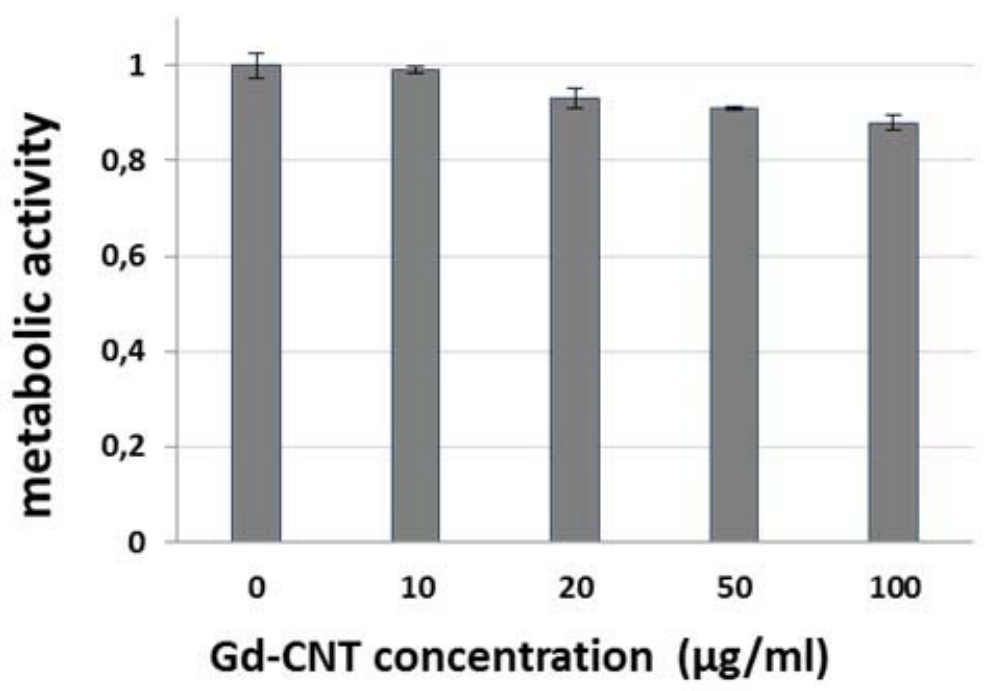

Figure S4: Metabolic activity of RAW 267.4 macrophages after $20 \mathrm{~h}$ incubation with GdCNTs 5 at concentrations of $10,20,50$ and $100 \mu \mathrm{g} / \mathrm{mL}$ normalized to that of control unexposed cells. 

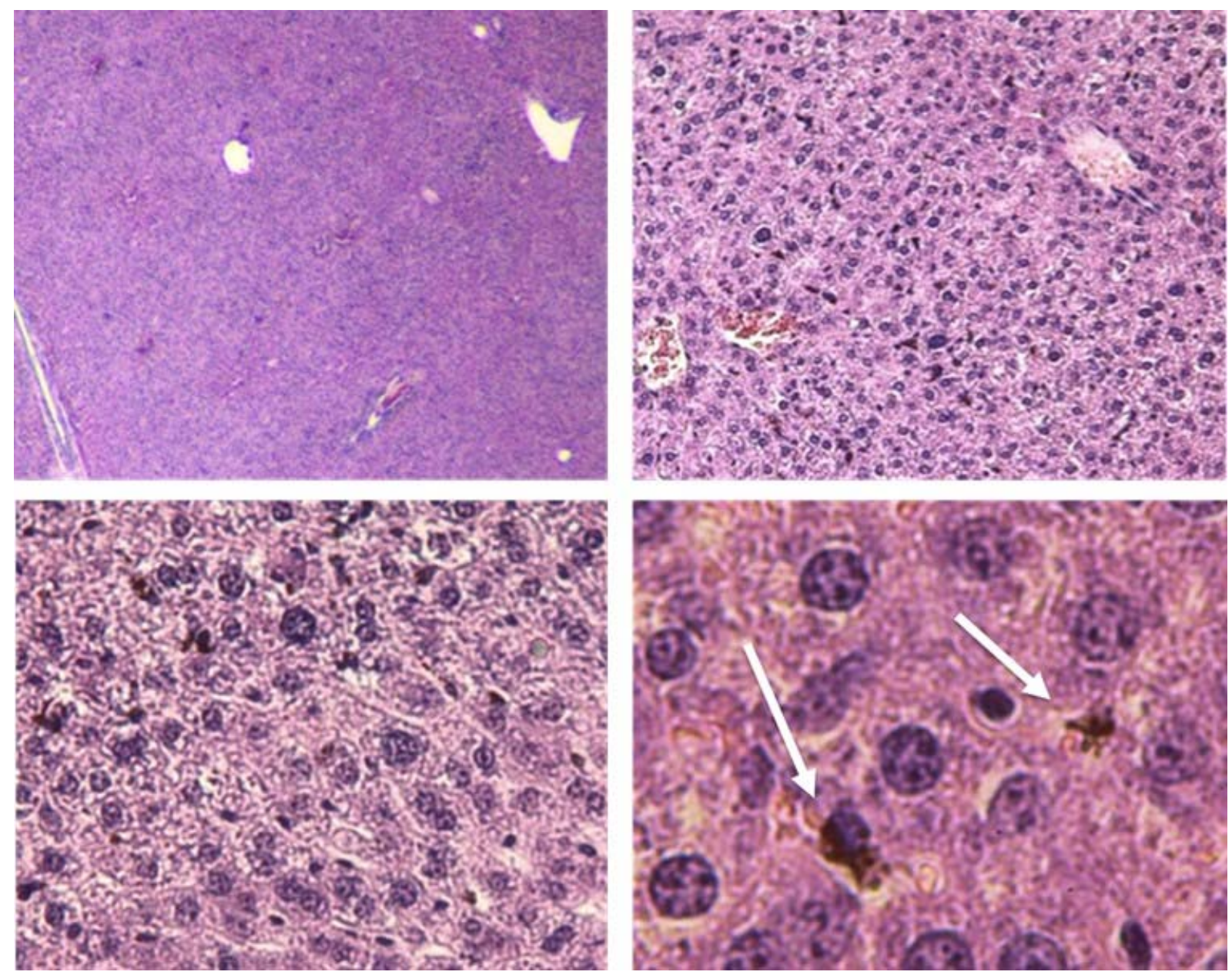

Figure S5: Histological specimens of liver after Hematoxylin- Eosin staining. Optical Magnification x2.5, x10, x20 and x63. White arrow points to Gd-CNT aggregates.
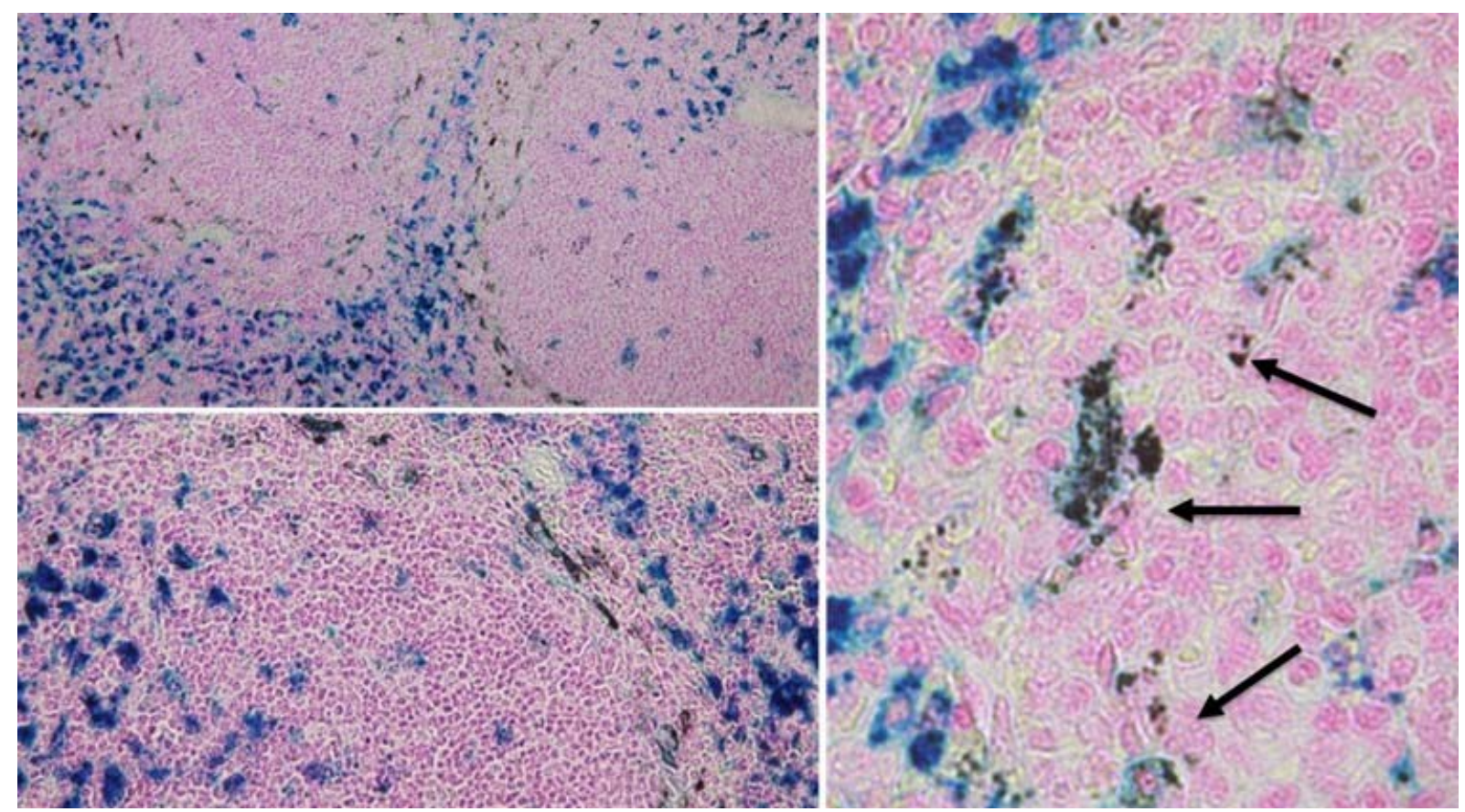

Figure S6: Histological specimens of spleen after Pearls and Nuclear Red staining, optical magnification, $\mathrm{x} 10, \mathrm{x} 20$ x 63 . Arrows point to Gd-CNT aggregates. 

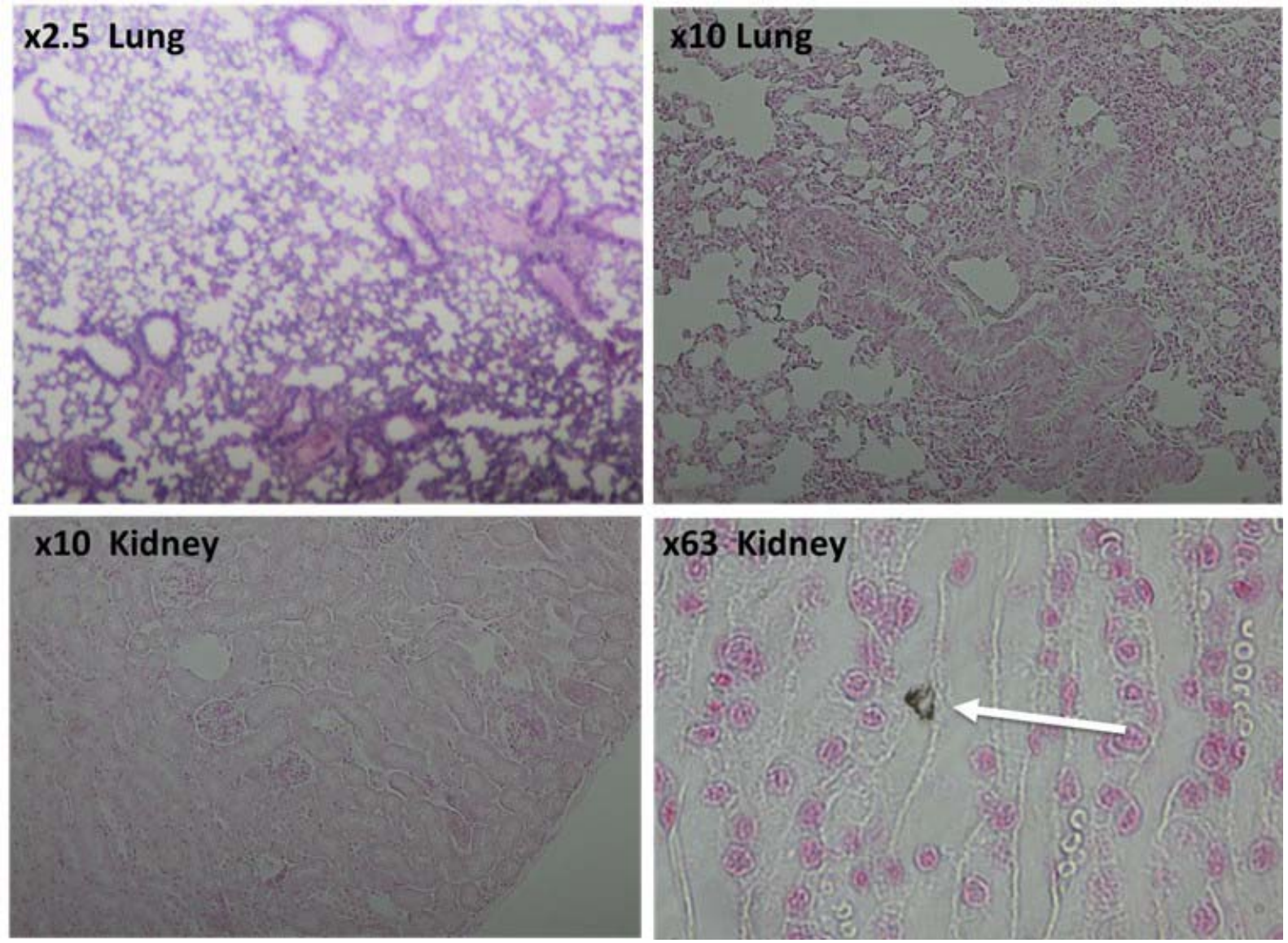

Figure S7. Histological micrographs of lung (nuclear red staining) and kidney (hematoxylineosin staining) at 6 hours following Gd-CNTs injection. Gd-CNTs are very rare both in lung and kidneys. White arrow points to a CNT aggregate in the kidney. (Optical magnification is indicated on the picture). 